\title{
A model for double notches and bifurcated components in radio profiles of pulsars and magnetars ${ }^{\star}$
}

\section{Evidence for the parallel acceleration maser in pulsar magnetosphere}

\author{
J. Dyks ${ }^{1}$, B. Rudak ${ }^{1}$, and Joanna M. Rankin ${ }^{2}$ \\ 1 Nicolaus Copernicus Astronomical Center, Toruń, Poland \\ e-mail: [jinx; bronek] @ncac . torun.pl \\ 2 Physics Department, University of Vermont, Burlington, VT 05405, USA
}

Received 30 October 2006 / Accepted 27 January 2007

\begin{abstract}
Context. Averaged pulse profiles of three nearby pulsars: B1929+10, J0437-4715 and B0950+08 exhibit unusual "double notches". These W-like looking features consist of two adjacent V-shaped dips that approach each other at increasing observation frequency $v_{\mathrm{obs}}$ roughly at a rate $\Delta \propto v_{\mathrm{obs}}^{-1 / 2}$, where $\Delta$ is the separation between the notches' minima.

Aims. We show that basic properties of the notches, namely their W-like look and the rate of their converging can be understood within a narrow class of models of coherent radio emission from pulsars: the free electron maser models based on coherent inverse Compton scattering of parallel oscillations of ambient electric field.

Methods. The observed properties of the pulsars imply that the Fourier spectrum of the wiggler-like oscillations is narrow and that the broad-band character of the radio emission reflects the width of the electron energy distribution.

Results. Such a model provides a natural explanation for the frequency-independent separation between the main pulse and interpulse of B0950+08 as well as for the lack of radius to frequency mapping in the conal-like emission of J0437-4715. The frequency behaviour of the main pulse in the profile of the first radio magnetar XTE J1810-197 can also be explained within this model.
\end{abstract}

Key words. stars: pulsars: general - stars: pulsars: individual: B1929+10 - stars: pulsars: individual: J0437-4715 -

stars: pulsars: individual: B0950+08 - stars: pulsars: individual: XTE J1810-197 - stars: radiation mechanisms: non-thermal

\section{Introduction}

Double notches are pairs of adjacent V-shaped dips observed in the averaged pulse profiles of three nearby pulsars: PSR B1929+10 (Rankin \& Rathnasree 1997), J0437-4715 (Navarro et al. 1997, hereafter NMSKB) and B0950+08 (McLaughlin \& Rankin 2004, hereafter MR04). The most striking property of the notches is that they have the intriguing "W" look: both notches have similar (if not identical) width $W$ which is approximately equal to their separation $\Delta$. The separation decreases for increasing observation frequency $v_{\mathrm{obs}}$ (NMSKB). The notches do not affect the degree of polarization nor its position angle.

Remarkably, the notches reside in weak and extended "pedestal" emission that exhibits many unusual properties: 1) it is observed far from the main pulse (MP); 2) covers long intervals of pulse longitude; and 3) shows three strange polarization characteristics: 3 a) it is very strongly linearly polarized ( $\$ 100 \%$ for B1929+10); 3b) the S-shaped position angle curve fitted to the pedestal emission only (with the MP excluded) is shifted leftward with respect to the MP (e.g. Everett \& Weisberg 2001). This is the opposite shift direction than expected for outward emission from a rotating magnetosphere (Blaskiewicz et al. $1991)$; 3c) the behaviour of the position angle is not very different from the predictions of the rotating vector model (RVM, Radhakrishnan \& Cooke 1969), which is puzzling because of 1)

* Appendices are only available in electronic form at http://www. aanda.org and 2), The properties 1) and 2) suggest that the pedestal radio emission may originate from locations in the magnetosphere that have little to do with the standard radio emission region. The closed field line region or extremely large altitudes, comparable to, or larger than, $R_{\mathrm{lc}}$ are not excluded. In the case of J0437-4715 the notches are located in a wing of a seemingly conal component. However, the conal-like components in J0437-4715 also exhibit some special properties, e.g. they do not follow the radius to frequency mapping. Inferences of this paper refer to this special pedestal and conal emission and should probably be not extended to all known emission components, e.g. the core components.

Existing models of double notches interpret them as a double eclipse of an extended emission region by a single absorber. The doubleness of such eclipse is caused by combined effects of differential (altitude-dependent) aberration and propagation time delays within the spatially extended emission region (Wright 2004). The absorber/eclipter may corotate in outer parts of pulsar magnetosphere (Wright) or remain stationary at the center of the magnetosphere (Dyks et al. 2005a, hereafter DFSRZ).

The models based on the differential special relativistic effects suffer from two main problems: 1) They provide no obvious reason for the $\mathrm{W}$ shape of the notches. Our preliminary, simplified calculations of pulse profiles for one version of such model (with the pulsar as the eclipter, DFSRZ) failed to produce the $\mathrm{W}$ shape. 2) The large radial extent of the emission region in these models and the strong caustic effects associated with the mechanism of the double eclipse should lead to rapid, 
complicated variations of position angle and strong depolarization (Dyks et al. 2004a). None of them is observed (in J0437-4715 they must be caused by interaction of orthogonal polarization modes). The simple property that $W \simeq \Delta$ is an extremely strong constraint on any physical model of the notches, and our main goal was to devise one that fulfills this requirement. In Sect. 2 we present the observed properties of double notches of B1929+10 and compare it to the other pulsars. In Sect. 3 we describe a general principle of our model and the numerical code used to calculate modelled shapes of notches. Section 4 describes how the look of modelled notches depends on various model parameters. Section 5 furnishes our model with physics and compares it with the observations.

\section{The double notches of PSR B1929+10}

Pulsar B1929+10 is a very useful object for studying the double notches because: 1) its notches are located far from the other strong emission components (MP, interpulse) and 2) the emission is strongly linearly polarized ( $\$ 100 \%$, Rankin \& Rathnasree 1997). Both properties ensure that the pedestal emission within and around the notches is not contaminated by contributions from different emission regions. In this section we report new observations of B1929+10 performed by one of us (JR) at Arecibo Observatory at frequencies $327 \mathrm{MHz}, 1.17 \mathrm{GHz}$, and $1.5 \mathrm{GHz}$ with the respective bandwidths of 25,100 and $200 \mathrm{MHz}$.

Figure 1a presents the averaged pulse profile of B1929+10 at $327 \mathrm{MHz}$. The double notches are visible at $\phi=103.5^{\circ}$. It will become clear below that a particularly constraining parameter for theoretical models is the depth $D_{\mathrm{n}}$ of the notches. Unfortunately, this quantity is also the most difficult to determine from observations because it depends on the amount of flux received from the pulsar at the lowest (dimmest) point of its pulse profile. This "unpulsed emission" was detected in B1929+10 via phase-resolved interferometric observations at $408 \mathrm{MHz}$ (Perry \& Lyne 1985, hereafter PL85).

To account for the depth measurement problem Fig. 1a represents the profile in a few different ways: The middle line (thin) is represented in the usual way, with the zero of the $y$-axis at the level $I_{\min }$ of the lowest place in the pulse profile, which we take as an average of data within the phase range $(-90,-80)$. For the top line we assumed that $I_{\min }=\left(1.5 \times 10^{-3} \pm 1.5 \times 10^{-4}\right) I_{\max }$, i.e. we take the same ratio of $I_{\min } / I_{\max }$ at $327 \mathrm{MHz}$ as observed by PL85 at $408 \mathrm{MHz}$. The error bar at $\phi=90^{\circ}$ marks the $2 \sigma$ error of the baseline level from PL85. The actual error may be larger if $I_{\min } / I_{\max }$ is strongly frequency dependent. The top two lines are multiplied by a normalization factor that sets the maximum observed flux $I_{\max }$ at 250 (beyond the figure) to reveal the shape of the weak pedestal emission. For the third, bottom pulse profile $I_{\max }=1$ and $I_{\min } / I_{\max }=1.5 \times 10^{-3}$. The upper two curves illustrate how strongly the derived depth of the notches depends on the baseline level: the tiny shift of the baseline from zero up to $1.5 \times 10^{-3} I_{\max }$ decreases $D_{\mathrm{n}}$ from nearly $40 \%$ (middle curve) down to $20 \%$ (top). Interestingly, the flux of the pedestal emission increases roughly linearly with time (angle) near the notches.

The 327-MHz pulse profile consists of two observations (12238 and 18835 single pulses long). A comparison of averaged pulse profiles for these two pulse sequences suggests that the relative intensity of the various weak structures in the pedestal may vary in time. The values of $D_{\mathrm{n}}$ derived for these two observations are equal to $17 \%$ and $24 \%$ (assuming $I_{\min } / I_{\max }$ from PL85). It is
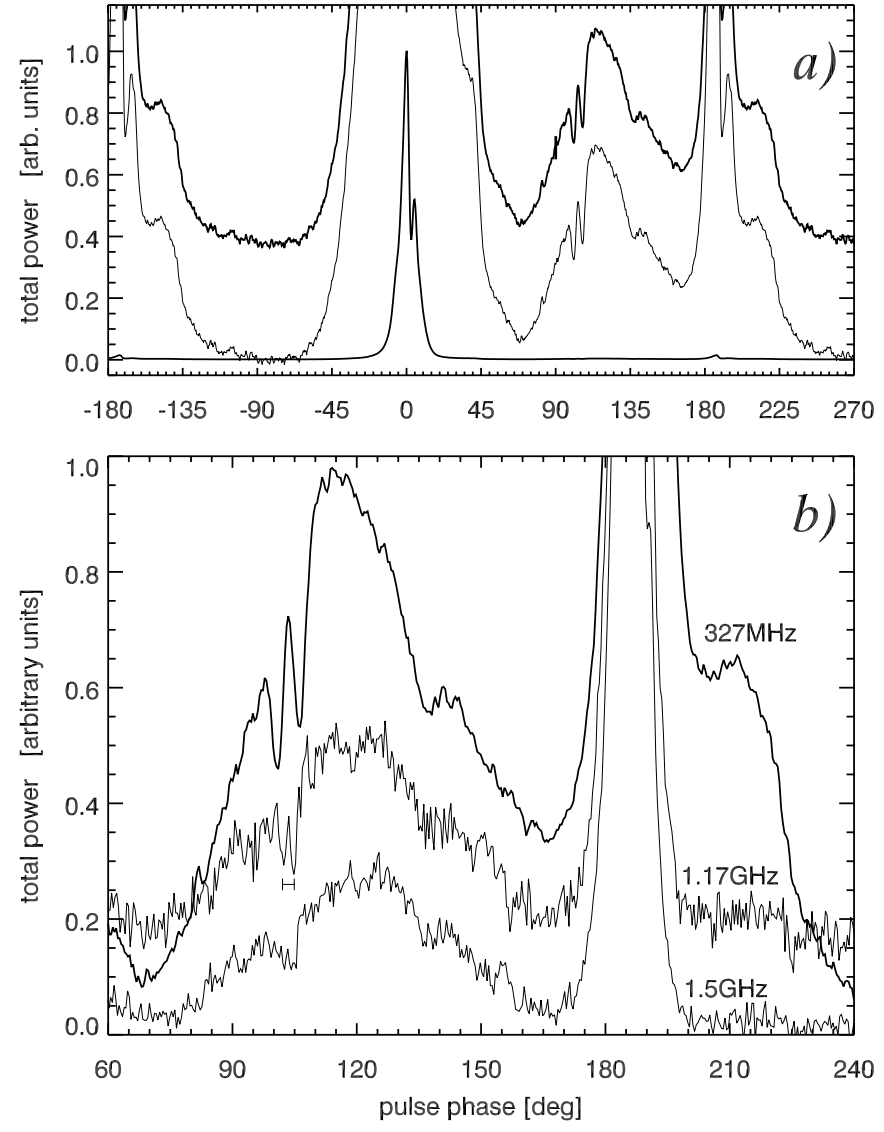

Fig. 1. Averaged pulse profiles of B1929+10 (all lines present the total flux). a) Three representations of the $327-\mathrm{MHz}$ profile that show how the baseline level affects the depth $D_{\mathrm{n}}$ of the notches. The top line has the minimum recorded flux $I_{\min }$ set at $0.0015 I_{\max }$ which results in $D_{\mathrm{n}} \simeq$ $20 \%$. The notches are much deeper $\left(D_{\mathrm{n}} \simeq 37 \%\right)$ if the profile is shown in normal way (thin middle line with $I_{\min }=0$ ). For both these profiles $I_{\max }=250$. The profile at the bottom has $I_{\max }=1$ and $I_{\min }=0.0015 I_{\max }$. b) Double notches at three different frequencies $v_{\text {obs. }}$. The horizontal bar below the notches at $1.17 \mathrm{GHz}$ marks the separation predicted by Eq. (4) for $\Delta_{327 \mathrm{MHz}}=5.36^{\circ}$. The phase alignment is such that the MP maxima at 1.17 and $1.5 \mathrm{GHz}$ lag the MP maximum at $327 \mathrm{MHz}$ by $0.8^{\circ}$. Different vertical scales and baseline levels were used at different $v_{\mathrm{obs}}$ for viewing purposes.

hard to tell whether this is caused by scintillation or intrinsic variability.

Figure 1b shows the frequency evolution of a part of the profile. The notches approach each other at $1.17 \mathrm{GHz}$ (middle curve) and seem to be merged into a single feature at $1.5 \mathrm{GHz}$ (bottom). Note that each of the profiles in Fig. 1b has undergone different linear transformation to fit a single plotting box (no meaning should be attached to the depth of the notches). The profiles were phase-aligned in such a way that the MP maximum of the $327-\mathrm{MHz}$ profile in our Fig. 1b precedes in phase the MP maximum of the $1.4-\mathrm{GHz}$ profile by $\Delta \phi_{\mathrm{MP}} \simeq 0.8^{\circ}$. Similar misalignment of the MP maxima can be discerned in published figures that present time-aligned profiles at different frequencies. From Fig. 2 of Kuzmin et al. (1998), that was obtained for $\mathrm{DM}=3.18 \mathrm{pc} \mathrm{cm}^{-3}$, one can estimate $\Delta \phi_{\mathrm{MP}} \simeq 1.3^{\circ}$. In Fig. 12 of Hankins \& Rankin (2006) $\Delta \phi_{\mathrm{MP}} \simeq 0.3^{\circ}$ for $\mathrm{DM}=3.176 \mathrm{pc} \mathrm{cm}^{-3}$. Interestingly, the shift of $0.8^{\circ}$ puts into perfect alignment both the notches and the maximum of the interpulse. Given that the shape of the MP changes strongly 


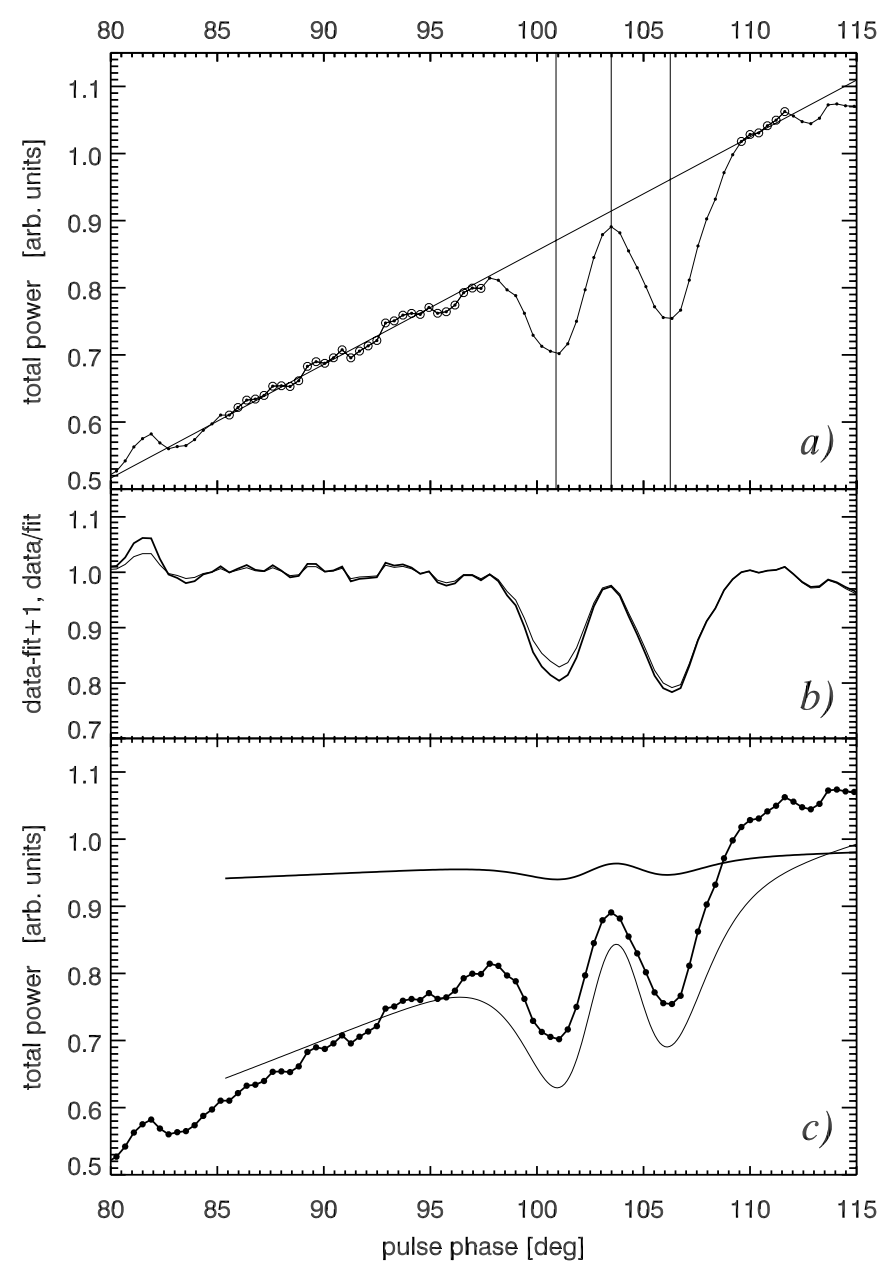

Fig. 2. A zoom into the $327 \mathrm{MHz}$ notches of B1929+10 (expanded part of the top line in Fig. 1a). The circled points in a) have been used to fit the linear variations of flux around the notches. We measure the depth of notches $D_{\mathrm{n}}$ along the outer vertical lines; the separation $\Delta$ is the horizontal distance between them. b) The notches with the linear trend removed. The thin line shows data - fit +1 ; the thick line presents the ratio data/fit. The leading notch is slightly less deep than the trailing one. c) One of model profiles (thick solid) obtained for the $\boldsymbol{a} \| \boldsymbol{v}$ case with $\gamma=10$ plotted over the data. The modelled notches are some $\sim 10$ times shallower than the observed ones. The thin solid line presents the same model result after a linear rescaling of the y-axis, which makes the modelled notches quite similar to the data.

between 0.4 and $1.4 \mathrm{GHz}$, it is reasonable to assume that the phase location of the MP maximum in B1929+10 is frequency dependent whereas the location of notches is fixed. Position of double notches definitely does not depend on frequency in J0437-4715 (NMSKB) nor in B0950+08 (MR04). All existing observations of notches are then consistent with their location in pulse profiles being frequency-independent. A physical explanation for this will be proposed in Sect. 5 .

Figure 2 presents a zoom into the same $327-\mathrm{MHz}$ notches as shown in Fig. 1a (top curve). Note that their appearance (especially the sharpness) is very sensitive to the assumed range on both the vertical and horizontal axis. Panel 2 a presents a linear fit to the marked data points around the notches (circles). The depths $D_{\mathrm{n}}$ are measured between this fit and the notches' minima as marked with the outer vertical lines. The result is $D_{\mathrm{n}}^{1}=(20.2 \pm 1) \%$ for the leading notch and $D_{\mathrm{n}}^{\mathrm{t}}=(21.8 \pm 1) \%$ for the trailing one (when expressed in percent, the depth of a given notch is normalized by the value of the linear fit at the phase at which the notch has the minimum; $2 \sigma$ errors are used throughout this paper). In Fig. $2 b$ the linear fit is subtracted from the data in two ways: the thin line represents data - fit +1 , whereas the thick line shows the ratio data/fit. In either case, the leading notch (located in the weaker emission) is shallower than the trailing notch in the stronger emission. The asymmetry holds at higher $v_{\text {obs }}$. With $\phi=0$ set at the MP maximum, the notches are located at $\phi_{1}=100.9 \pm 0.07^{\circ}$ and $\phi_{t}=106.25 \pm 0.06^{\circ}$, i.e. they are separated by $5.36 \pm 0.1^{\circ}$. The center of $\mathrm{W}$ lags the MP by $103.5 \pm 0.1^{\circ}$.

An important feature that strongly constrains the theoretical models is that the maximum between the double notches (at the center of the "W") nearly (but not exactly) reaches the level interpolated with the linear fit. The data point marked in Fig. 2a with the central vertical line $\left(\phi=103.5^{\circ}\right)$ is $2.6 \%$ below the fit level, i.e. it misses the fit by $\sim 10 \%$ of $D_{\mathrm{n}}$.

\subsection{Notches in other pulsars}

In the millisecond pulsar J0437-4715 the emission with notches is stronger $\left(\sim 8 \%\right.$ of $I_{\max }$ at $\left.438 \mathrm{MHz}\right)$ and a bit less polarized $(\sim 70 \%)$ than in the case of B1929+10. The notches actually seem to be carved out of the trailing wing of a prominent trailing component in the main pulse (see figures in NMSKB). At sub$\mathrm{GHz}$ frequencies the top of the trailing component is split into two maxima separated by a single dip/notch. This bifurcated top is probably bright enough (at least at $v_{\mathrm{obs}} \lesssim 400 \mathrm{MHz}$ ) to be studied on a single pulse basis (see Sect. 5.2). We are not aware of a baseline flux measurement for this object, so that only the upper limit of $\sim 50 \%$ for the notch depth can be estimated. If the unpulsed flux from this pulsar is comparable to a few percent of $I_{\max }$ then the actual $D_{\mathrm{n}}$ is considerably smaller.

As in the case of B1929+10, the double notches in J0437-4715 approach each other for increasing frequency $v_{\text {obs }}$, though they are still separable in the high quality $1.5-\mathrm{GHz}$ pulse profile of NMSKB. The split at the top of the trailing component also gets narrower at increasing frequency and looks nearly like a single feature at $1.5 \mathrm{GHz}$

On the theoretical side, the pulse profile of J0437-4715 may be more difficult to model because its magnetosphere is much smaller $\left(P=5.76 \mathrm{~ms}, R_{\mathrm{lc}}=27.5 \times 10^{6} \mathrm{~cm}\right)$ than that of B1929+10 $\left(P=0.2265 \mathrm{~s}, R_{\mathrm{lc}}=1080 \times 10^{6} \mathrm{~cm}\right)$ so that the radio emission has a larger probability of being affected by general relativistic effects.

Observations of B0950+08 performed by MR04 at $430 \mathrm{MHz}$ give $D_{\mathrm{n}} \sim 10-16 \%$ and PL85 report negligible amounts of unpulsed emission for this object at a similar frequency. However, the notches of B0950+08 are located close to the MP, in a region probably contaminated by several types of emission, as the low polarization degree suggests. It is therefore not excluded that the actual $D_{\mathrm{n}}$ is larger than given above. On the other hand, the weak emission components in B0950+08 have been reported to vary on a time scale of several days (MR04), and it is hard to tell at what stage of this variability the observations of PL85 were done. The notches of B0950+08 seem fairly blurred at high frequencies (see inset in Fig. 3 in MR04).

\section{The model}

The model postulates an emission region with large extent in the rotational azimuth $\phi$ and rotational colatitude $\theta(\Delta \phi \gg \Delta$ and $\Delta \theta \gg \Delta$ ). For simplicity, we assume the emission is produced at a fixed altitude (see Sect. 4.4 for more comments on emission 

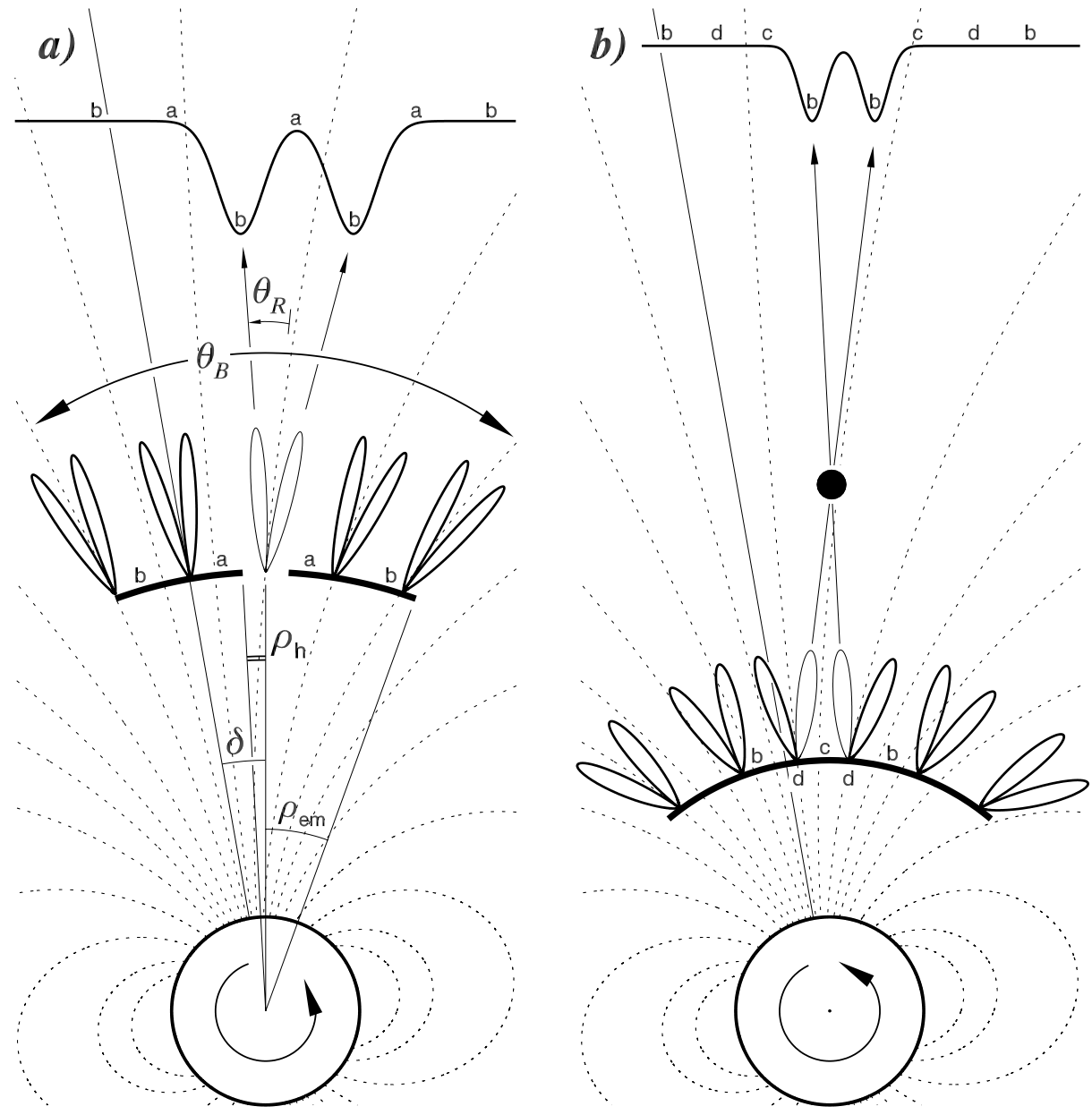

Fig. 3. The principle of our model. The plane of rotational equator for a pulsar with dipole inclination $\alpha=$ $90^{\circ}$ chosen for simplicity is shown. The thick solid arc is the crossection through a two-dimensional emission region. Crossections through several hollow cone elementary beams of radio emission are indicated. The observer is located near the plane of the figure. The dotted curves mark the dipolar magnetic field lines. In a) the notches form because of the hole in the emission region. In b) they are created by a single absorber above the region (marked with bullet). The small letters help to associate different points of emission region with pulse phase at which the radiation emitted from them is observed.

region). The key assumption is that on a microscopic scale the region radiates a hollow cone beam along a local direction of magnetic field $\boldsymbol{B}$. A single hole in the emission region produces a cone of reduced emission, as shown in Fig. 3a. The notches can be observed when the observer's line of sight cuts through the cone. A single absorber between an observer and the source of radio emission will act in a similar way (Fig. 3b). The part of the emission region that is "half-hidden" from the observer has the shape of a ring. Its crossection is marked with "d" in Fig. $3 b$.

There are at least two basic reasons for which the elementary emission beam can have the hollow cone shape:

1) Electrons may be accelerated along their velocity $\boldsymbol{v} \| \boldsymbol{a}$ and the radio emission observed in the pedestal can be due to this acceleration (Melrose 1978; Kunzl et al. 1998; Schopper et al. 2002; Levinson et al. 2005). In this case the opening angle of the elementary beam $2 \theta_{\mathrm{R}} \sim 1 / \gamma$, where $\gamma \sim 10$ is the Lorentz factor of the electrons. More exactly the received power has a maximum at the angle

$\theta_{\mathrm{R}}=\arccos \left[\frac{\left(1+24 \beta^{2}\right)^{1 / 2}-1}{4 \beta}\right] \approx \sqrt{\frac{1}{5}} \cdot \frac{1}{\gamma} \approx 0.4472 \cdot \frac{1}{\gamma},(1)$

where $\beta=v / c$ is the electron speed in units of the speed of light and the approximation holds for $\gamma \gg 1$. In this version of the model the observed separation of the double notches is:

$\Delta \approx \frac{2 \theta_{\mathrm{R}}}{\sin \zeta}=\frac{2}{\sqrt{5} \gamma \sin \zeta}=\frac{0.8944}{\gamma \sin \zeta} \approx \frac{1}{\gamma}$ where $\zeta$ is the viewing angle measured from the rotation axis, the factor $1 / \sin \zeta$ takes into account the "not a great circle" effect (e.g. Dyks et al. 2004b), and the latter approximation holds with accuracy better than $10 \%$ for any angle $52^{\circ}<$ $\zeta<128^{\circ}$. The equation is approximate in that it does not take into account the angle $b$ between the hole axis and the line of sight. Numerical results of the next section tell us that this "hole impact angle" does not affect $\Delta$ as long as the notches have the W-like shape (see Fig. 4d). Equation (2) is valid for the case of the emission region with a hole (Fig. 3a). Note that according to Eq. (2) the observed magnitude of $\Delta \sim 2^{\circ}-6^{\circ}$ implies Lorentz factors of the order of 10 , which is a value estimated on independent grounds in pulsar models based on the parallel acceleration (e.g. the ALAE model of Melrose 1978 or CICS model of Schopper et al. 2002).

2) The elementary beam has the hollow cone shape also when high-energy electrons $(\gamma \gg 10)$ are accelerated perpendicularly $(\boldsymbol{a} \perp \boldsymbol{v})$ but most of them have non-negligible pitch angle $\psi \gg 1 / \gamma$ (see e.g. Fig. 6.5 in Rybicki \& Lightman 1979). In this case $\theta_{\mathrm{R}}=\psi_{\max }$, where $\psi_{\max }$ is the pitch angle for which the distribution of electrons $n_{\mathrm{e}}(\psi)$ has a maximum. The non-zero pitch angle requires low magnetic field, such as is present at large altitudes, comparable to $R_{\mathrm{lc}}$ (Lyubarski \& Petrova 1998; Malov \& Machabeli 2001; Petrova 2003; Harding et al. 2005) as well as at lower altitudes in the millisecond pulsars (e.g. J0437-4715). In spite of some positive features (see Appendix A), the pitch angle version of our model is less successful in reproducing the data and is disfavored in this paper. 


\subsection{The numerical code}

The numerical code to simulate the notches assumes: 1) specific geometry and size of the emission region; 2) specific geometry and size of the hole/fissure or absorber; 3 ) the structure of the magnetic field within the emission region, e.g. dipolar, radial (swept out by wind) or toroidal (sweepback); 4) the geometry of the elementary emission beam; in the case $\boldsymbol{v} \| \boldsymbol{a}$ it is calculated directly from the classical electrodynamics formulae (e.g. Eq. (4.101), in Rybicki \& Lightman 1979) for a selected value of $\gamma$ (we do not integrate over the electron-energy distribution). In the case of $\boldsymbol{v} \perp \boldsymbol{a}$ we assume the shape of the pitch angle distribution (e.g. $n_{\mathrm{e}} \propto \psi \exp \left[-\psi^{2} / \psi_{\max }^{2}\right]$, as in Epstein 1973), and integrate the single electron "pencil" beams of radiation over the pitch angle distribution to get the "elementary" emission beam for a fixed value of $\gamma$. Next, the emission region is divided into a large number of tiny fragments, each of which is equipped with its own elementary beam. An observer located at a viewing angle $\zeta$ is selected and a lightcurve is calculated by integration over the source for each pulse longitude (the place where the observer's line of sight enters the elementary beam is determined individually for each fragment of the source). The symmetry axis of the elementary beams is assumed to point along the local magnetic field $\boldsymbol{B}$ in the corotating frame. The aberration and propagation time delays are neglected because of the fixed emission altitude.

To integrate the received flux over the electron-energy distribution one needs to know how the coherent emissivity $\epsilon$ at a specific frequency $v_{\text {obs }}$ depends on the electron Lorentz factor $\gamma$. This depends on the radio emission/coherency mechanism that actually works in pulsar magnetosphere. Even for a specific emission mechanism, the dependence of $\epsilon(\gamma)$ at a fixed $v_{\text {obs }}$ may be completely different for different values of $v_{\text {obs }}$. Let us take the noncoherent curvature radiation (that certainly cannot be responsible for the emission with notches) as an example. Well below the maximum in the curvature spectrum $\left(v \ll v_{\mathrm{CR}}\right)$ the emissivity does not depend on $\gamma$, whereas above the maximum (in the region of the exponential cut-off) it becomes extremely sensitive to it: $\epsilon_{\mathrm{CR}} \propto \exp \left[-\right.$ const. $\left.\cdot \gamma^{-3}\right] \cdot \gamma^{-1 / 2}$. For a weak dependence of $\epsilon(\gamma)$ the integration over the electron energy distribution could significantly blur the notches. It is therefore very important that in the model of the parallel acceleration maser based on the coherent scattering of the "wiggler-like" field, the emissivity is tightly focused around $v_{\mathrm{obs}} \sim \gamma^{2} v_{\mathrm{wgl}}$ (see left panel of Fig. 4, in Schopper et al. 2002), where $v_{\mathrm{wgl}}$ is the frequency of oscillations of the ambient electric field.

The angular distance $\theta_{\mathrm{h}}$ of the hole from the rotation axis and the dipole inclination $\alpha$ have little effect on the shape of notches, except from blowing them up by the factor $1 / \sin \theta_{\mathrm{h}}$. Therefore, in all cases presented in this paper we assume that $\theta_{\mathrm{h}}=\alpha=90^{\circ}$. The emission region in all simulations of notches is a fragment of a sphere that extends significantly both in $\phi$ and $\theta$ direction $\left(\Delta \phi=\Delta \theta=10 \theta_{\mathrm{R}}\right)$.

\section{Numerical results}

We have modelled numerous configurations with different hole geometries, absorber, $\boldsymbol{B}$-field structure and with various pitch angle distributions $n_{\mathrm{e}}(\psi)$. In general, results are sensitive to bulk geometry/topology of the $\boldsymbol{B}$-field and emission/absorption region. In the pitch angle case they also depend on the form of function $n_{\mathrm{e}}(\psi)$.

\subsection{The parallel acceleration case - a hole in the dipolar $B$-field}

Figure 4 presents results obtained for a circular hole in the dipolar magnetic field (the case $\boldsymbol{v} \| \boldsymbol{a}$ with $\gamma=10$ ). In 4 a the hole is centered at the dipole axis $(\delta=0)$, and its angular radius $\rho_{\mathrm{h}}$ increases from 0.1 to $3.3 \theta_{\mathrm{R}}$ (top to bottom). The observer was located at a "hole impact angle" $b \equiv \theta_{\mathrm{h}}-\zeta=90^{\circ}-\zeta=0$, i.e. the line of sight sweeps through the hole's center. For $\rho_{\mathrm{h}}<0.3 \theta_{\mathrm{R}}$ the notches have the "W"-like shape similar to the observed one, with the flux at the center of the "W" at nearly the same level as beyond the notches. An important difference between these cases and observations is that the depth of the modeled notches does not exceed a few percent, in comparison with a few tens of percent tentatively derived from observations. This is a major concern for the present version of the model and will be discussed below. For a larger $\rho_{\mathrm{h}}$ the central flux drops down until a single wide "notch" with an initially flat bottom appears at $\rho_{\mathrm{h}} \sim 0.7 \theta_{\mathrm{R}}$.

In $4 \mathrm{~b}$ the top four curves from $4 \mathrm{a}$ are replotted with a stretched $y$-axis. This shows how sensitive the appearance of the notches is to the selected viewing method. The strong stretch of the $y$-axis produces the fall-off of flux for the increasing $|\phi|$. This occurs because the dipolar magnetic field lines are more spreaded at larger magnetic colatitudes $\theta_{\mathrm{m}}$ measured from the dipole axis (so that a smaller number of lines are pointing towards a unit solid angle at increasing $\theta_{\mathrm{m}}$ whereas the emissivity of the emission region is assumed to be uniform per unit surface). Note that a similar stretch of the $y$-axis may be unknowlingly applied to plots that show the observed pulse profiles if the baseline level has been overestimated (Fig. 1a).

Figure $4 \mathrm{c}$ shows the effect of a non-axisymmetric location of the hole: the dipole axis has been rotated by the angle $\delta=$ $30^{\circ}$ with respect to the line connecting the centers of the hole and star (see Fig. 3a). According to the basics of dipolar field geometry, the notches moved to $\phi \approx 0.5 \delta$. The displacement of the hole off the dipole axis results in moderate asymmetry of the notches' shape. The approximately linear decrease of flux around the notches is caused by the increasing spread of $\boldsymbol{B}$-field lines.

Figure $4 \mathrm{~d}$ presents the shape of notches for different viewing angles $\zeta=\theta_{\mathrm{h}}-b$ and the other parameters $\left(\rho_{\mathrm{h}}, \gamma\right)$ the same as in the second-from-top case in panel c. For $b<0.3 \theta_{\mathrm{R}}$ the "W"-like notches can be observed. For larger $b$ their shape evolves into a single dip. Interestingly, the separation between the notches practically does not depend on $b$ as long as $b<0.7 \theta_{\mathrm{R}}$. The separation starts to decrease only for $b \lesssim \theta_{\mathrm{R}}$, i.e. when the flux at the W center becomes nearly as low as at the notches' minima. This property allows us to ignore the ratio of $b / \theta_{\mathrm{R}}$ in Eq. (2) as well as in the discussion of the frequency dependence of $\Delta$ in Sect. 5 below (note that $b / \theta_{\mathrm{R}}$ can change with $v_{\mathrm{obs}}$ for a single object viewed by a fixed observer).

For the increasing $\gamma=10,20,40$ the modelled notches approach each other, the flux at the $\mathrm{W}$ center decreases, and they finally merge into a single feature (solid lines in panels e and $\mathrm{f}$ of Fig. 4). This resembles the behaviour observed at different frequencies (Fig. 1b). It can be directly interpreted within our model if radiation at higher frequencies is mainly generated by electrons with larger Lorentz factor $\gamma$. The depth of the modelled feature increases when the notches merge. This does not seem to happen in the data (Fig. 1b) although the baseline level at the 1.17 and $1.5 \mathrm{GHz}$ is not known. As we show in the next section, however, the interpretation is supported if our geometric idea is supplied with the physics of parallel acceleration maser. 

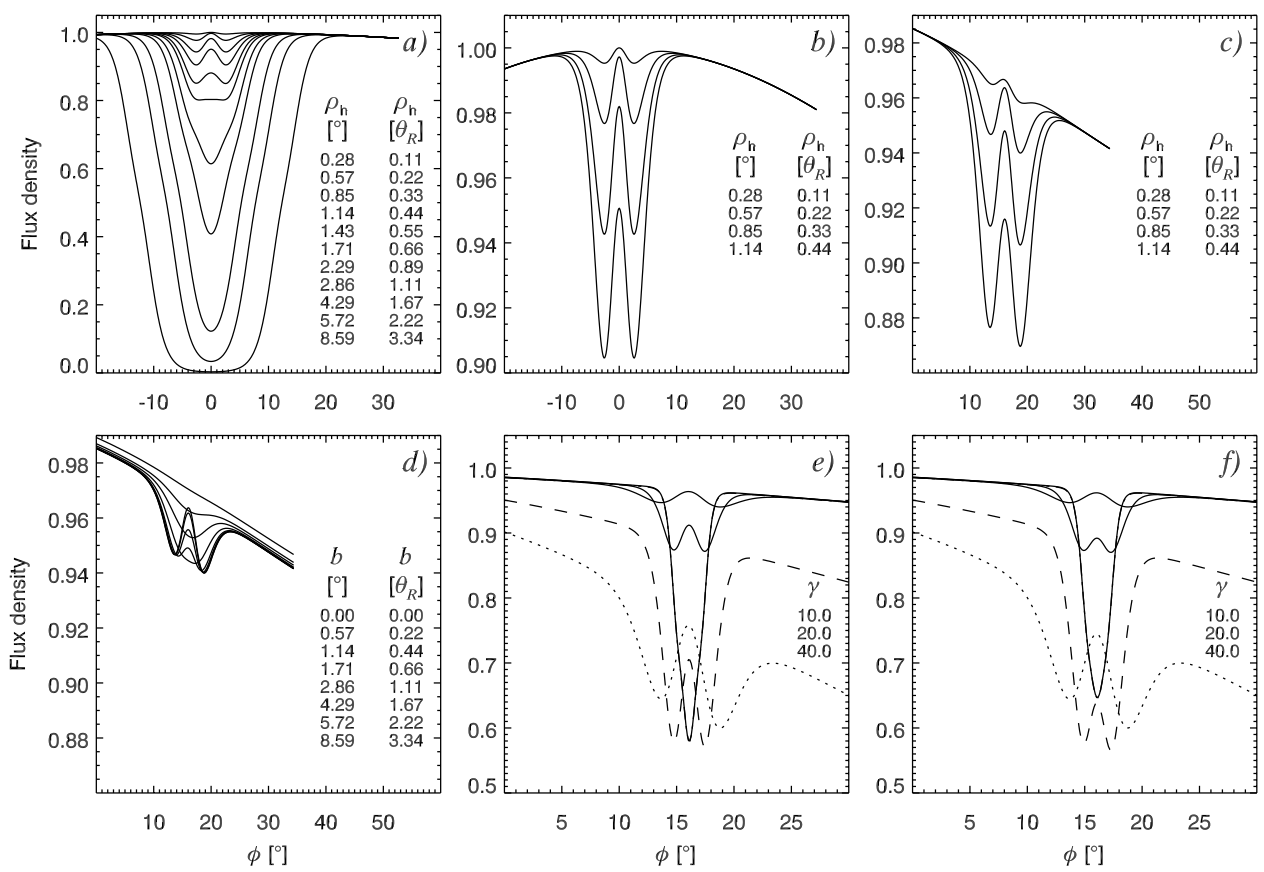

Fig. 4. Notches modelled for a hole in the dipolar $\boldsymbol{B}$-field for the case $\boldsymbol{a} \| \boldsymbol{v}$. a) The effect of increasing hole radius $\rho_{\mathrm{h}}$ for $\gamma=10, b=90^{\circ}-\zeta=0$ and $\delta=0$, i.e. with the hole centered at the dipole axis (top to bottom). b) Top four curves from a) replotted with a rescaled vertical axis. c) Same as b) but with the hole located off-axis $\left(\delta=30^{\circ}\right)$. d) The effect of increasing "impact" angle $b$ for $\rho_{\mathrm{h}}=0.22 \theta_{\mathrm{R}}$ and $\gamma=10$. e) The effect of increasing $\gamma$ for $\rho_{\mathrm{h}}=0.57^{\circ}$ and $b=0$ (solid curves). The lines for $\gamma=10$ and 20 were linearly transformed and replotted as the dotted and dashed curves, respectively. f) Same as e) but for $b=0.57^{\circ}$. Unlike in many figures that show observed pulse profiles, in all cases shown with solid lines the zero of $y$-axes corresponds to "no flux".
As we show in Appendix A one can increase the depth of notches up to $\sim 20 \%$ in the pitch angle scenario by varying the function $n_{\mathrm{e}}(\psi)$. However, this is usually associated with considerable deformation of their shape. Modelled notches with a depth and shape reasonably similar to the observed one can be obtained only after application of artificial "linear rescaling" of model lightcurves. Figure $2 \mathrm{c}$ shows the result of such transformation for the parallel acceleration case. The thick line with dots shows the observed $327-\mathrm{MHz}$ notches of B1929+10 with the baseline level corrected according to the $410-\mathrm{MHz}$ measurement of PL85. The thick solid line with the very shallow notches is the second-from-top result from Fig. $4 \mathrm{c}$ after a mirror reflection and horizontal shift has been applied to it (with no rescaling in any axis). The thin solid line in Fig. $2 c$ is the same model result after a linear rescaling of $y$ axis. This exercise is to check whether the shape of the rescaled notches in the $\boldsymbol{a} \| \boldsymbol{v}$ case bears any resemblance to the observed ones. The agreement is moderate: the outer sides of the modelled notches are less steep than the observed one.

\subsection{Geometry of the hole/absorber}

The geometry of the hole or absorber is the next important factor that affects the shape and depth of the notches. We have considered a few cases that produce deeper notches. However, this has always been accompanied by the deformation of their shape. An obvious way to make the notches deeper is to extend the hole in the direction perpendicular to the rotational azimuth. This, however, strongly decreases the flux at the W center (Fig. 5, thin line). Much the same result is obtained when an elongated absorber (dense plasma stream?) is placed above the emission region. A desperate way to avoid the decreased central flux is to assume an opaque thin wall/fin protruding from the emission region upward. Such configuration can produce very deep notches with the central flux unaffected (if the fin is thin). However, the notches have a shape different than observed $(\Delta \ll W)$ and become extremely asymmetric if the fin is located asymmetrically in the dipolar magnetic field (off the dipole axis). Though the fin cannot be responsible for the observed notches (and it would be hard to justify its origin) this case shows that a geometric configuration that gives deep notches with the unaffected central flux is possible.

\subsection{Magnetic field geometry}

In addition to the dipolar $\boldsymbol{B}$ we have considered the radial and toroidal magnetic field. The radial field has similar geometry to the dipolar field near the dipole axis and it produced similar results. The toroidal field $\left(B_{x}=\cos \phi, B_{y}=\sin \phi, B_{z}=0\right)$ was considered as the idealized model of the swept-back, near- $R_{\mathrm{lc}}$ magnetic field. The direction of such $\boldsymbol{B}$ at a fixed azimuth is independent of $\theta$ so that all parts of the extended source that are located at the meridian selected by the line of sight contribute equally to the observed flux. This tends to decrease $D_{\mathrm{n}}$.

\subsection{Emitter's geometry}

Our present choice of the emitter was dictated by two facts: 1) the weak pedestal emission that contains the notches covers a very large range of pulse phase. This implies that the angular extent of the emitter must be large in $\phi$, and consequently in $\theta$. A negligible extent in $\theta$ would make the detection of the emission less probable, whereas the extended emission components seem to be quite common among the nearest and brightest pulsars. From the inspection of profiles in the EPN pulsar data base one can learn that within the distance of the furthest pulsar with notches (B1929+10 at $0.36 \mathrm{kpc}$ ) only $50 \%$ of pulsars in the ATNF catalogue (Manchester et al. 2005) has the radio emission limited to a narrow range of pulse phase (few tens of degrees), and some objects have really wild pulse profiles (e.g. J2124-3358, Manchester \& Han 2004); 2) the PA curve for the pedestal emission closely follows the curve of Komesaroff (1970) which probably implies that the source is not very extended in the radial direction $\left(\Delta r \ll R_{\mathrm{lc}}\right)$. The fixed emission altitude that we have assumed can be considered a good approximation for any region with the radial extent that is negligible in comparison with other relevant length scales. These include the scale $\rho_{\text {crv }} \Delta$ on which the magnetic field changes direction 


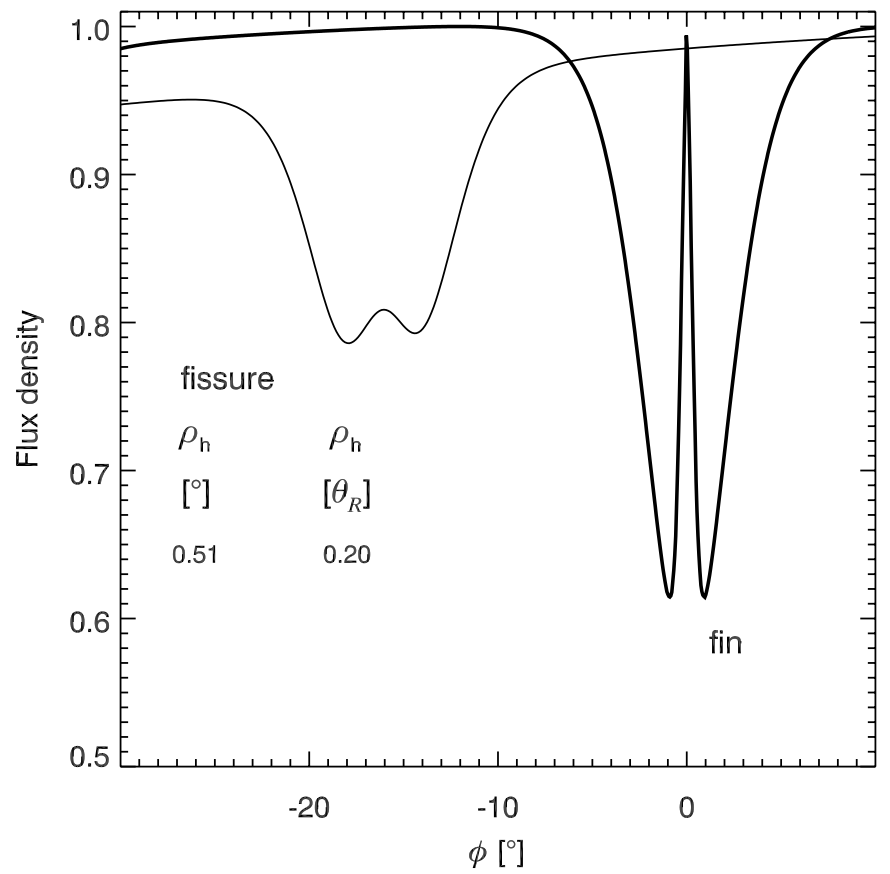

Fig. 5. Modelled double notches for a meridionally extended fissure (thin line) and for a meridionally extended fin that protrudes vertically upward from the emission region (thick line). The notches have large depth but their shapes do not resemble the observed one.

by angle $\Delta$, where $\rho_{\text {crv }}$ is the radius of curvature of magnetic field lines. The other scale is the one on which the electron plasma frequency drops significantly (for a power-law decrease of plasma density it is of the order of the emission height $r$ ). Both these length scales are comparable. Strong, fixed frequency radio emission from a "too large" range of altitudes would probably make the notches less distinct. The only emitter that we have managed to consider so far (i.e. the part of spherical surface shown in Fig. 3) surely does not exhaust all the possible configurations that can produce the extended pedestal and have a reasonable detection probability. While simulations for other emitters are being considered, we turn to the observational consequences of physical aspects of the model.

\section{Model versus observations}

Based on a large number of numerical results, we find that a few key features of model notches agree well with the observations:

1. whenever the sightline cuts through the cone of reduced emission the calculated profiles tend to have two minima/ notches;

2. both notches have similar depth;

3. the width of the notches tends to be equal to their separation.

The list can be considerably extended if our simple idea is supplemented with the physics of a specific version of a free electron maser:

4. If the emission at a higher frequency $v_{\mathrm{obs}}$ is mainly generated by electrons with larger Lorentz factors, then the elementary emission beam is narrower at larger $v_{\text {obs }}$ which directly implies that the separation of notches is smaller, as is indeed observed. In the case of $\boldsymbol{a} \| \boldsymbol{v}$, the elementary beam becomes narrower simply because of Eq. (1).
According to some models of pulsars (e.g. Melrose 1978; Schopper et al. 2002; Levinson et al. 2005) the ambient electric field and plasma density within emission region(s) in pulsar magnetosphere tend to oscillate with a frequency $v_{\mathrm{wgl}}$ that can result either from global magnetospheric electrodynamics (e.g. Sturrock 1971; Levinson et al. 2005) or can be locally excited by streams of energetic electrons penetrating the ambient plasma (e.g. Schopper et al. 2002). Hereafter we call this frequency a "wiggler" frequency by a rough analogy with the laboratory free electron laser (FEL) ${ }^{1}$. In the free electron maser models of coherent radio emission from pulsars the observed radio emission can be considered as the Compton scattered (and blueshifted) wiggler frequency $v_{\mathrm{wgl}}$ :

$v_{\mathrm{obs}} \simeq \gamma^{2} v_{\mathrm{wgl}}$.

What are the spectra of $\gamma$ and $v_{\mathrm{wgl}}$ is a difficult question, but one simple and seemingly natural possibility is that the electron energy distribution is much broader than the Fourier spectrum of the wiggler oscillations, i.e. $v_{\mathrm{wgl}} \sim$ const. in Eq. (3) for a given object. In this case different $v_{\text {obs }}$ can be associated with the inverse-Compton scattering of the same wiggler frequency $v_{\mathrm{wgl}}$ by electrons with different Lorentz factors. This implies (from Eqs. (2) and (3)) that the separation of double structures should decrease with frequency according to

$\Delta \simeq \sqrt{\frac{4}{5}} \frac{1}{\sin \zeta}\left(\frac{\nu_{\mathrm{wgl}}}{v_{\mathrm{obs}}}\right)^{1 / 2} \propto v_{\mathrm{obs}}^{-1 / 2}$.

This dependence is in good agreement with the observed behaviour of the notches at different frequencies. Figure 6 presents $\Delta$ as a function of $v_{\mathrm{obs}}$ as derived from the data on J0437-4715 (NMSKB, Jenet et al. 1998) and B1929+10 (MR04; this work). All points except from the two diamonds (J0437 at $1380 \mathrm{MHz}$ ) have $2 \sigma$ errors marked. The locations of the diamonds have been estimated from Fig. 1 in Jenet et al. (1998). The vertical bars at these points have the ad hoc length of $\pm 10 \%$ of $\Delta$.

5. The model expressed by Eq. (4) assumes that different radio frequencies are generated by electrons that have different energy but occupy the same emission region. This is in clear contrast to the traditional view according to which different $v_{\mathrm{obs}}$ originate from different altitudes with different electron plasma frequency, i.e. are associated with variations of $v_{\mathrm{wgl}}$ in Eq. (4). Our model predicts then that locations of emission components in averaged pulse profiles should not change with $v_{\mathrm{obs}}$. This is in perfect agreement with the frequency independence of the separation between the interpulse (IP) and main pulse in B0950+08 (Hankins $\&$ Cordes 1981). The interpulse is connected with a bridge of low intensity emission with leading components of the MP. All the components likely have the same origin because they exhibit similar long term variability (cf. Figs. 1 and 2 in MR04) as well as similar (low) intensities of single pulses (Nowakowski 2003). Therefore, it is probable that the entire stretch of emission that includes the IP and the "notched bump" ahead of MP is generated by the inverse Compton parallel acceleration maser. Thus, we are surprised to realize that the issue of whether the MP-IP separation does

1 Unlike in the device, in the models mentioned above the wiggler field is oscillating parallel to local $\boldsymbol{B}$-field and electron velocity. Perpendicular wiggler oscillations have also been considered in the context of pulsars (see Fung \& Kuijpers 2004). 


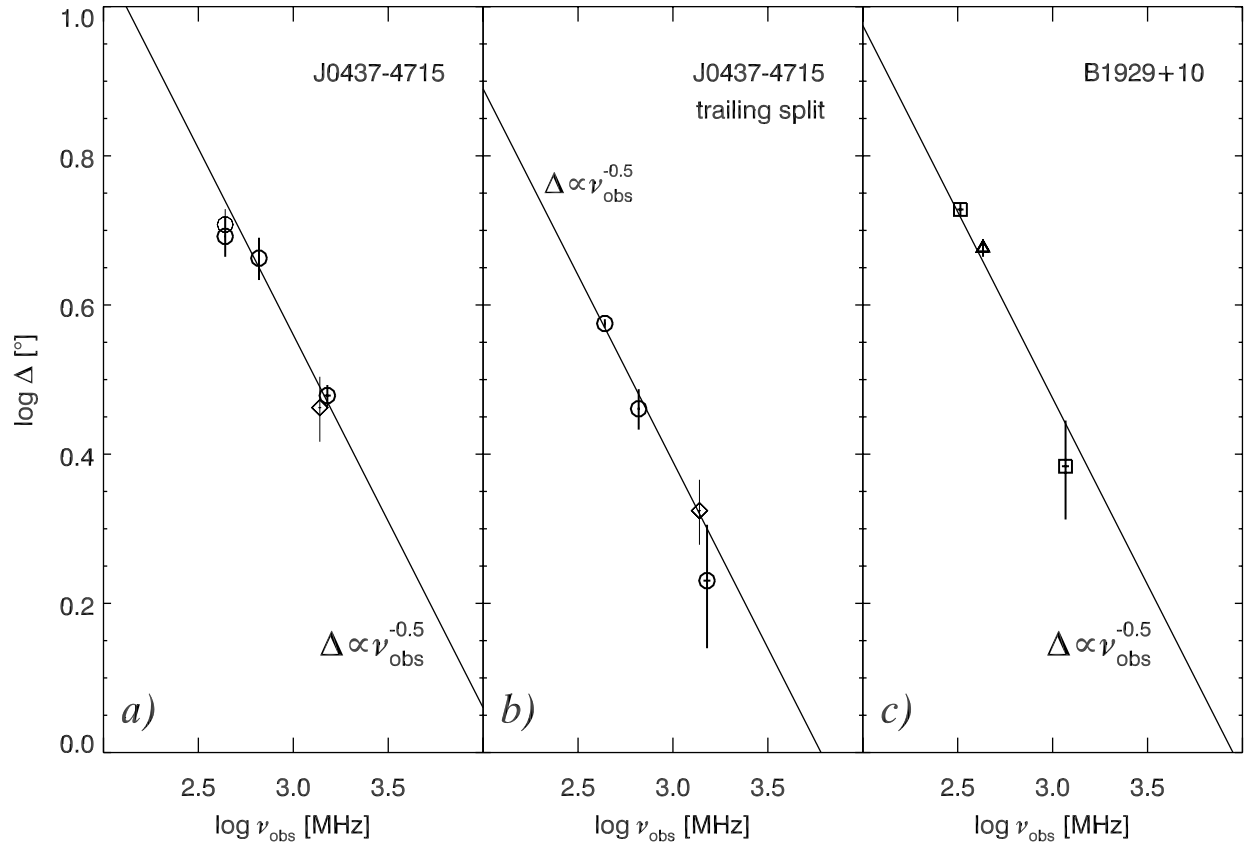

Fig. 6. Observed separation $\Delta$ of double notches as a function of frequency $v_{\mathrm{obs}}$ for J0437-4715 a) and $\mathrm{B} 1929+10 \mathrm{c})$. The middle panel is for the trailing bifurcated component in J0437-4715. The error bars are $2 \sigma$ for all points except from the two diamonds (J0437-4715 at $1380 \mathrm{MHz}$ ) which are equipped with bars of length $\pm 10 \%$ of $\Delta$. The straight lines mark the relation $\Delta \propto v_{\mathrm{obs}}^{-1 / 2}$ predicted by Eq. (4). The light circle for the lowest frequency point of J0437-4715 refers to the separation of notches in the averaged profile of linearly polarized radiation $\mathrm{L}$. The data are from NMSKB (circles), Jenet et al. (1998) (diamonds), MR04 (triangle) and this work (squares). (or does not) depend on $v_{\mathrm{obs}}$ has nothing to do with the problem of whether we see one pole or two poles. However, since some parallel acceleration models have inherently built-in two-directional emission (e.g. Levinson et al. 2005), it is still reasonable to interpret the structure formed by the IP, bridge and MP in B0950 within the two-directional emitter scenario (Dyks et al. 2005b; Fowler \& Wright 1982; Cheng \& Ruderman 1977). This speculation needs to be verified by detailed modelling.

6. For the same reason (broad band radio emission caused by electron energy distribution) the conal-like components in J0437-4715 do not exhibit any sign of radius-to-frequency mapping over a very wide frequency range (McConnell et al. 1996; NMSKB). McConnell et al. (1996) report an intrinsic low-frequency spectral turnover around $\sim 100 \mathrm{MHz}$ which can be associated within our model with a lower boundary of the electron energy distribution at $\gamma_{\min } \simeq$ $6 / \sin \zeta$. It is worth mentioning that below $v_{\mathrm{obs}} \simeq v_{\mathrm{wgl}} \gamma_{\min }^{2}$ the relation $\Delta \propto v_{\text {obs }}^{-1 / 2}$ is not expected to hold.

7. The oscillations of ambient $\boldsymbol{E}$ may be far from stable on long time scales. Evolution or disturbances of the oscillation frequency (broadening of the oscillation spectrum) would smear the notches. This is qualitatively consistent with the observed temporal variations of the shape of double notches (MR04; did Phillips 1990 see the notches? - see Fig. 2 therein, and comments in Rankin \& Rathnasree 1997 or MR04).

8. If the emission is indeed caused by the parallel acceleration, there should be a chance (provided that the macrosopic geometry of the emission region is suitable) to see the elementary hollow cone in emission. There is indeed a bifurcated emission component seen in the same interval of emission that contains the double notches in J0437-4715 (see Fig. 1 in NMSKB). Bifurcated components observed in some pulsars (e.g., also for the millisecond PSR J1012+5307, see Fig. 1 in Xilouris et al. 1998; or for the radio magnetar XTE J1810-197, see Fig. 1 in Camilo et al. 2006) may result from the cut of our line of sight through emission from such a region. In Fig. 7 we present a modelled lightcurve for an elongated emission region that was thin in the azimuth direction $\left(\Delta \phi=0.3 \theta_{\mathrm{R}}\right)$ but very elongated in colatitude $(\Delta \theta=$ $\left.10 \theta_{\mathrm{R}}\right)$. This configuration can be considered to be a zeroth order approximation of a trailing side of a ring centered at the dipole axis. One can see that the large meridional extent of the emission region does not blur the hollow cone shape of the elementary beam completely. The central panel of Fig. 6 shows that the separation between the maxima of the bifurcated component in J0437-4715 also follow the relation $\Delta \propto v_{\mathrm{obs}}^{-1 / 2}$.

9. The only known radio magnetar - XTE J1810-197 - exhibits radio emission that is variable on several long timescales (days and months, Camilo et al. 2006, 2007) which makes it distinct from normal radio pulsars. The shape of its averaged pulse profile became extremely unstable in July 2006 (Camilo et al. 2007). Several months before, however, the object exhibited emission with many features similar to those of the "notched emission" of J0437-4715. Its radiation was highly polarized $(89 \pm 5 \%$ at $8.4 \mathrm{GHz}$, Camilo et al. 2006), the main pulse was bifurcated and connected smoothly to an extended emission component at low $v_{\text {obs }}$ (see Fig. 1 in Camilo et al. 2006, note that the phase alignment of the profiles at different $v_{\mathrm{obs}}$ is arbitrary). The frequency behaviour of the MP is unlike the normal radius to frequency mapping: instead of the evolution from a single component at high $v_{\mathrm{obs}}$ to the well separated two conal components at low $v_{\text {obs }}$ the MP becomes broader but retains its basic shape through the very wide frequency band of $0.7-42 \mathrm{GHz}$. We argue here that the frequency evolution results from the broad electron energy spectrum (Eq. (4) with $v_{\mathrm{wgl}} \sim$ const.). In Fig. 8 we show that the separation between the maxima in the MP approach each other at a rate reasonably consistent with the $v_{\text {obs }}^{-1 / 2}$ law at $v_{\text {obs }} \gtrsim 1 \mathrm{GHz}$. The vertical error bars have magnitude of $2 \sigma$.

At sub-GHz frequencies $\Delta$ ceases to increase, probably because of a 'boundary effect' caused by a low energy limit $\gamma_{\min }$ in the electron energy distribution. The sub-GHz radiation may form a low energy end of the radio spectrum with $v_{\mathrm{obs}} \lesssim v_{\mathrm{wgl}} \gamma_{\min }^{2}$. The three flux-density measurements 


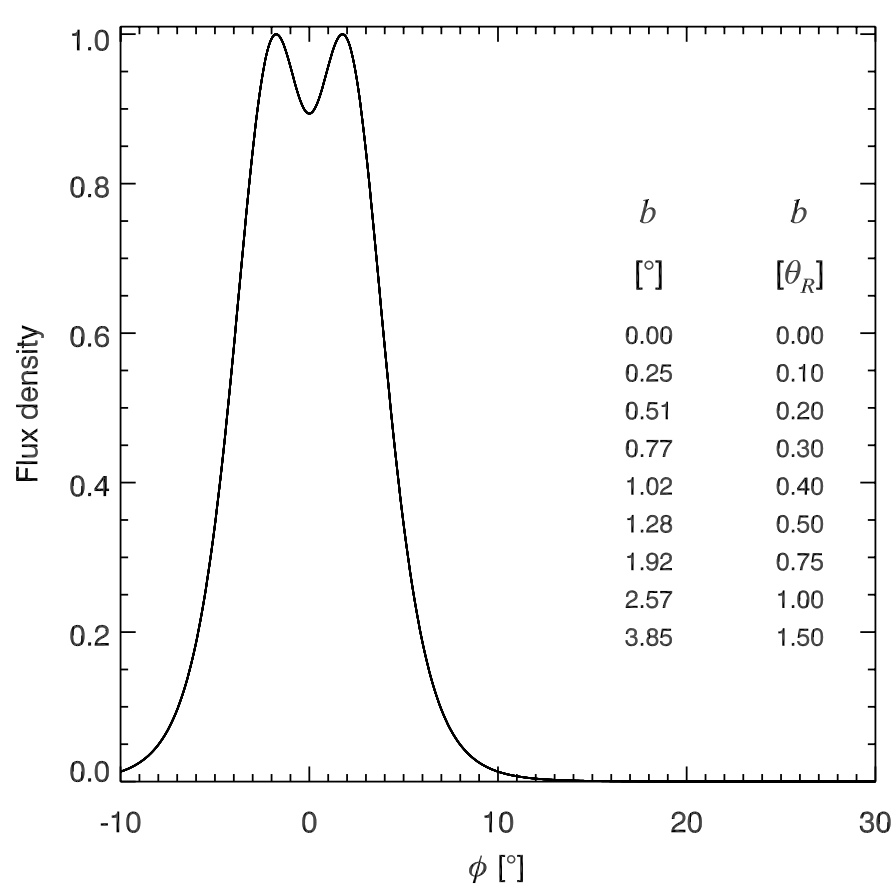

Fig. 7. Modelled pulse profile for an emission region considerably extended in the rotational colatitude $\theta\left(\Delta \theta \gg \theta_{\mathrm{R}}, \Delta \phi<\theta_{\mathrm{R}}\right)$. The elementary emission beam had a shape of the hollow cone for the parallel acceleration case with $\gamma=10$. The hollowness is revealed by the $\sim 10 \%$ dip at the top of the pulse. The result does not depend on the viewing angle $\zeta$.

between 0.69 and $2.9 \mathrm{GHz}$ done on MJD 53850.9 (Table 1 of Camilo et al. 2006) are indeed consistent with a harder spectrum $\left(S_{v} \propto v_{\mathrm{obs}}^{0}\right)$ than measured at higher frequencies $\left(S_{v} \propto v_{\text {obs }}^{-0.5}\right.$ between 1.4 and $\left.19 \mathrm{GHz}\right)$ on MJD 53857 .

10. Contrary to other models of double notches (Wright 2004; DFSRZ) the emission region considered here does not have to be radially extended (it may extend moderately or not at all). The large depolarization and extremely complicated position-angle curve are characteristic features of regions with large radial extension (Dyks et al. 2004a) and are not observed in the pedestal emission. The observed polarization properties of the pedestal emission thus seem to be more consistent with the present model.

\subsection{Predicted periodicity of microstructure}

The microstructure observed in pulsars exhibits typical timescales or quasi-periodicities (e.g., Cordes et al. 1990; Lange et al. 1998) that used to be interpreted within the FEL type models of pulsars as a direct result of the wiggler oscillations and are used to estimate the value of the Lorentz factor in these models. Since in our model $\gamma$ can be independently estimated from the notches' separation, the model can be verified by checking whether the typical timescale of microstructure is equal to

$\tau_{\mathrm{wgl}}=\frac{1}{v_{\mathrm{wgl}}} \simeq \frac{\gamma^{2}}{v_{\mathrm{obs}}}=\frac{0.8}{v_{\mathrm{obs}} \Delta^{2} \sin ^{2} \zeta}$.

Had it been possible to observe both the double notches and the periodic microstructure with the timescale $\tau_{\mathrm{wgl}}$, one could use the above equation to constrain $\zeta$.

The width of the wiggler spectrum allowed by the model can be assessed as follows: if we assume that the notches get blurred

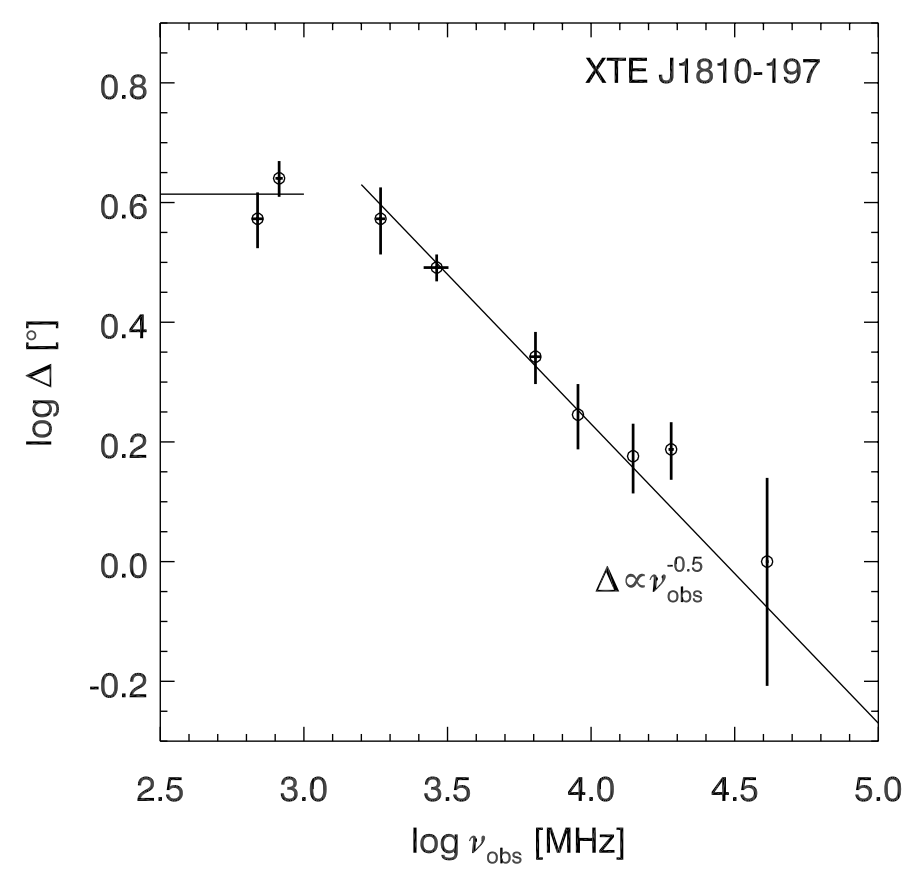

Fig. 8. Observed separation $\Delta$ of the maxima in the main pulse of the first known radio magnetar XTE J1810-197 as a function of observation frequency $v_{\text {obs }}$. The errors of $\Delta$ have magnitude of $2 \sigma$. The horizontal bars (too short to be visible for most points) show a bandwidth. The straight lines mark the relation $\Delta \propto v_{\mathrm{obs}}^{-1 / 2}$ given by Eq. (4). The data are from Camilo et al. (2006).

when $1 / \gamma$ changes "significantly" (say by a factor of 2 ), then the spectrum of $v_{\mathrm{wgl}}$ cannot extend by a factor larger than 4 (since $\left.1 / \gamma \propto v_{\mathrm{wgl}}^{1 / 2}\right)$. This rough estimate applies only to the part of magnetosphere that produces detectable radio emission.

For B1929+10 we observe $\Delta \simeq 5.3^{\circ}$ at $327 \mathrm{MHz}$, i.e. $\gamma=$ $9.7 / \sin \zeta$ and $\tau_{\mathrm{wgl}}=0.28 \mu \mathrm{s} \sin ^{-2} \zeta$ which requires single pulse observations with sub-microsecond time resolution. Given the weakness of the pedestal emission in B1929+10 this observation can be unfeasible even for SKA. In the other pulsars, however, the emission with notches (or with double emission features) is much brighter (between a few and 30 percent of $\left.I_{\max }\right)$ and should be easier to observe. For B0950+08 one gets $\Delta \simeq 5.3^{\circ}$ at $430 \mathrm{MHz}$ (MR04) so that $\gamma \simeq 9.7 / \sin \zeta$ and $\tau_{\mathrm{wgl}}=0.22 \mu \mathrm{s} \sin ^{-2} \zeta$. The maxima of the bifurcated trailing component in the pulse profile of J0437-4715 are separated by some $3.9^{\circ}$ at $438 \mathrm{MHz}(\mathrm{NMSKB})$. This gives $\gamma \simeq 13 / \sin \zeta$ and $\tau_{\mathrm{wgl}} \simeq 0.4 \mu \mathrm{s} \sin ^{-2} \zeta=1.6 \mu \mathrm{s}\left(\sin 30^{\circ} / \sin \zeta\right)^{-2}$. Attempts to reveal the periodicity of microstructure in J0437-4715 have been done at two widely separated frequencies $(327 \mathrm{MHz}$, Ables et al. 1997; and $1380 \mathrm{MHz}$, Jenet et al. 1998) with apparently conflicting results. The periodicity of $22 \mu$ s reported by Ables et al. would be consistent with Eq. (5) for a very small viewing angle $\zeta \sim 8^{\circ}$ that we consider somewhat extreme. Jenet et al. (1998) report no microstructure periodicity down to $80 \mathrm{~ns}$ but their single pulse signal could have been dominated by the bright core component with little contribution from the "notched emission" that is weak at $1380 \mathrm{MHz}$.

The "absorber version" of our model allows for a range of possible microstructure periodicity, because the counterpart of Eq. (5) becomes additionally dependent on geometric parameters of the system (e.g. the distance of the absorber from the emission region, the curvature of the region, etc.). Whereas the 
$v_{\mathrm{obs}}^{-1 / 2}$ dependence can appear in this case under some conditions (e.g. in radial $\boldsymbol{B}$-field), a specific $\Delta$ translates in this scenario into a larger opening angle $\theta_{\mathrm{R}}$ and smaller $\gamma$ (see Fig. 3). The expected $\tau_{\mathrm{wgl}}$ are therefore smaller than in the case shown in Fig. 3a.

\subsection{Single pulse visibility of double notches and emission cones}

Our numerical simulations assumed that the radio emissivity was uniform and steady throughout the entire emission region, because we were modelling double features in averaged pulse profiles. A natural question is whether the double notches can be observed in single pulse emission. A related question is whether the hollow cone shape of the elementary radio beam can be directly observed as pairs of emission features in the instantanenous (single pulse) radio emission. The simple answer to these questions is "no" - the notches that are pronounced in an average profile should not be seen as an absorption feature in the single pulse data. The hollowness of the emission cone that can be evident in the averaged pulse profile (Fig. 7) cannot be recognized in single pulses either. The reason is that the instantaneous emissivity within the emission region is very non-uniform and variable as suggested by features observed on a variety of timescales shorter than $P$ (e.g. Weltevrede et al. 2006; Edwards et al. 2003; Johnston \& Romani 2002; Cairns et al. 2004) and as is normally assumed in the models of drifting subpulses (Ruderman $\&$ Sutherland 1975; Wright 2003).

The detailed discussion is deferred to Appendix B. Here we only mention the timescale relevant to the problem to isolate some limiting cases. To simplify the analysis we assume the equatorial geometry illustrated in Fig. $1\left(\alpha=\zeta=90^{\circ}\right)$. Let us consider a single bunch of electrons (a spark) associated with a single hollow cone of radio emission of opening angle $2 \theta_{\mathrm{R}}$. Let us initially assume that the bunch is "frozen", i.e. it does not move in the corotating frame and its emission cone does not evolve. If the leading side of its cone is directed towards the observer at some moment $t_{1}$ then a considerable period of time

$\Delta t_{\mathrm{rot}} \simeq \frac{2 \theta_{\mathrm{R}}}{\Omega} \simeq \frac{P \Delta}{2 \pi} \simeq 7 \times 10^{-5} \mathrm{~s} \frac{P}{5 \mathrm{~ms}} \frac{\Delta}{5^{\circ}}$

must pass before the rotation of the magnetosphere directs the trailing side of the cone towards the observer, at moment $t_{\mathrm{t}}$. To detect both sides of the cone the fictious frozen bunch would have to survive for $\Delta t \gtrsim \Delta t_{\text {rot }} \sim 7 \times 10^{-5}-3 \times 10^{-3} \mathrm{~s}$, where the range corresponds to $P \simeq 5-250 \mathrm{~ms}$ observed among the pulsars with notches. The corresponding light travel distance is $\Delta r_{\text {rot }}=c \Delta t_{\text {rot }} \sim(2-105) \times 10^{6} \mathrm{~cm}$, i.e. of the order of, or much larger than the neutron star radius $R_{\mathrm{NS}}$. In Appendix B we discuss two types of limitations that make the observability of the notches in single pulses improbable/impossible: one is purely geometrical and associated with the non-uniform illumination of the radially thin emission region (slowly drifting bright spots that do not evolve on timescales shorter than $\Delta t_{\text {rot }}$ nor do they move relativistically in any direction). The other one is due to special relativistic kinematics and refers to a realistic situation of fast moving (outflowing) bunches of electrons that evolve on a timescale $\Delta t \ll \Delta t_{\text {rot }}$ that is too short for a bunch to expose both sides of its elementary emission cone to the observer.

\section{Conclusions/discussion}

We conclude that a remarkable number of peculiar observational effects can be understood within a radio coherency model that is based on inverse-Compton scattering of a single "wiggler" frequency (or a narrow band of wiggler frequencies) by a broad energy distribution of electrons. The model explains the "W"-like shape of the notches, the bifurcated emission components, the convergence of these features at the rate $v_{\text {obs }}^{-1 / 2}$, the frequency independence of the separation between the IP and MP in B0950+08 as well as the lack of radius-to-frequency mapping in J0437-4715. The model performs reasonably well for the only known radio magnetar XTE J1810-197, which implies that the same mechanism of coherent radio emission is operating in objects with so wildly different surface magnetic fields as the magnetars and millisecond pulsars. This finding is consistent with the linear acceleration origin of radio emission, because the strength of $\boldsymbol{B}$-field is largely irrelevant for this mechanism (Rowe 1995).

There are many unsolved puzzles that remain and that need to be addressed in future. These are the depth of the notches, the macroscopic geometry of the emitter and hole/absorber, the nature of the hole, etc. They are closely interrelated so it may be worthwhile to address all of them simultaneously rather than treat them separately. The large depth of double notches could easily be produced if the coherent radio emission had occured at two small angles $(\sim 1 / \gamma)$ with respect to the plane of $\boldsymbol{B}$-field lines. When smeared along the $\boldsymbol{B}$-field lines this would result in a kind of two-planar emission geometry. The maser based on curvature drift (Zheleznyakov \& Shaposhnikov 1979; Luo \& Melrose 1992, hereafter LM92) offers this kind of emission geometry (see the bottom panel of Fig. 1b in LM92) but it predicts much weaker frequency dependence of $\Delta$ than observed ( $\Delta \propto v_{\text {obs }}^{-1 / 3}$ according to Eq. (9) of LM92). In the case of models based on parallel acceleration, part of the hollow cone beam near the $\boldsymbol{B}$-field line plane would have to get absorbed to obtain the two-planar emission geometry. An interesting question is whether the emission is outward or perhaps inward, as suggested by the leftward shift of the position-angle curve. It is worth emphasizing that two-directional emission is inherent in some of the parallel acceleration models (e.g. Levinson et al. 2005), although bulk of a magnetosphere of fast rotating pulsars (J0437-4715) has been estimated opaque for the backward emission (Luo \& Melrose 2006). An important issue is the altitude of the emission. The typical radio frequency expected in free electron maser models is proportional to electron plasma frequency and is too high in comparison with the observed $\sim 100 \mathrm{MHz}$ emission (Kunzl et al. 1998; Melrose 2000). This favors large emission distance $\left(r \sim R_{\mathrm{lc}}\right)$ which is consistent with the far-from-MP location of the pedestal emission (provided that B0950+08 or B1929+10 have large dipole inclinations, which seems probable). The strength of the guiding magnetic field required to stabilize parallel Langmuir waves is too low $\left(10^{3} \mathrm{G}\right.$ in Schopper et al. 2002) to constrain possible emission site. On the other hand, the nearly RVM shape of the position-angle curve (Rankin \& Rathnasree 1997) suggests low emission altitudes and these would require radiation from the closed field line region. The region is a place of copious pair production according to the outer gap model (Cheng et al. 1986; Hirotani et al. 2003; Takata et al. 2006; Wang et al. 1998, see Fig. 3 therein), which is quite successful in reproducing gammaray pulse profiles of pulsars (e.g. Romani \& Yadigaroglu 1995; Dyks \& Rudak 2003). The details of the macroscopic geometry of the system remain a puzzle, and we emphasize that the configurations shown in Fig. 3 may be very far from reality.

A big unsolved issue is what is the relation of the radio emission considered in this paper to the more normal (?) emission that can be classified within the scenario of core and conal beams 


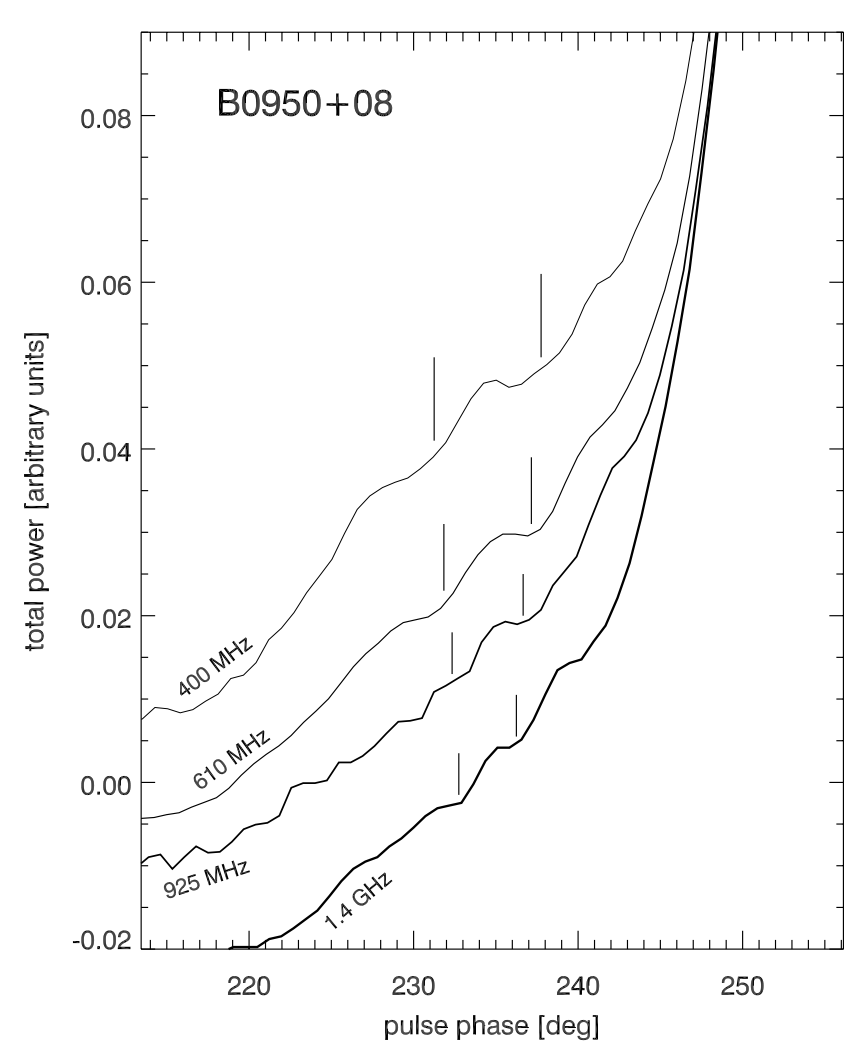

Fig. 9. A fragment of pulse profile of B0950+08 that shows the behaviour of double notches at increasing frequency (top to bottom). The data are from the European Pulsar Network base (Gould \& Lyne 1998). Pairs of vertical bars above each profile present the relation $\Delta=6.5^{\circ}\left(408 \mathrm{MHz} / v_{\text {obs }}\right)^{1 / 2}$ (Eq. (4)).

that exhibit nulling, drifting as well as the radius to frequency mapping. Can the RFM-exhibiting conals be interpreted within the same "parallel FEL" model, but dominated by variations of $v_{\mathrm{wgl}}$ in Eq. (3)? What is the origin of core emission?

The model proposed here can be tested observationally by searching for microstructure periodicities in the emission with notches or with bifurcated components (Eq. (5)). The expected timescale of the microstructure is of the order of $1 \mu \mathrm{s}$. Multifrequency observations of other objects at a high signal-tonoise ratio can provide further support for the relation $\Delta \propto v_{\mathrm{obs}}^{-1 / 2}$. Since there is some evidence of temporal evolution of double notches, their separation at a fixed frequency may in principle vary in time. Therefore, simultaneous multifrequency observations would be most preferred, although even the nonsimultaneous data in Figs. 6 and 8 proved successful in revealing the $\Delta\left(v_{\text {obs }}\right)$ relation. The millisecond pulsar $\mathrm{J} 1012+5307$ has a bifurcated component and is a very good candidate (see. Fig. 5 in Kramer et al. 1999). The double notches of B0950+08 present a difficult observing target (Fig. 9) and it is important to determine their multifrequency behaviour with better definition. The broad band approach can also tell us whether breaks in the relation $\Delta\left(v_{\text {obs }}\right)$ are associated with spectral breaks. A very important but difficult task is to precisely determine baseline levels for the pulsars with notches at different frequencies. This would provide the depths of notches that strongly constrain possible geometric configurations of the magnetospheric emitter.

Acknowledgements. This paper was inspired by the recent works of Geoffrey A. E. Wright (MNRAS 2003, 2004). J.D. thanks J. Borkowski, F. Camilo, Q. Luo, and D. Melrose for helpful comments. I am also grateful to R. Manchester and S. Ransom for the amazing pulsar data. We acknowledge the support of the KBN grant N203 017 31/2872 and NSF grants (JMR). Arecibo Observatory is operated by Cornell University under contract to the US National Science Foundation. Part of this research has made use of the data base of published pulse profiles maintained by the European Pulsar Network, available at: http://www.mpifr-bonn.mpg.de/pulsar/data/. We have also used the ATNF pulsar Catalogue (http://www . atnf.csiro. au/ research/pulsar/psrcat).

\section{References}

Ables, J. G., McConnell, D., Deshpande, A. A., \& Vivekanand, M. 1997, ApJ, $475, \mathrm{~L} 33$

Blaskiewicz, M., Cordes, J. M., \& Wasserman, I. 1991, ApJ, 370, 643

Cairns, I. H., Johnston, S., \& Das, P. 2004, MNRAS, 353, 270

Camilo, F., Ransom, S. M., Halpern, J. P., et al. 2006, Nature, 442, 892

Camilo, F., Cognard, I., Ransom, S. M., et al. 2007, ApJ, submitted [arXiv:astro-ph/0610685]

Cheng, A. F., \& Ruderman, M. 1977, ApJ, 216, 865

Cheng, K. S., Ho, C., \& Ruderman, M. A. 1986, ApJ, 300, 500

Cordes, J. M., Weisberg, J. M., \& Hankins, T. H. 1990, AJ, 100.2, 1882

Daugherty, J. K., \& Harding, A. K. 1983, ApJ, 273, 761

Dyks, J., \& Rudak, B. 2003, ApJ, 598, 1201

Dyks, J., Harding, A. K., \& Rudak, B. 2004a, ApJ, 606, 1125

Dyks, J., Rudak, B., \& Harding, A. K. 2004b, ApJ, 607, 939

Dyks, J., Frạckowiak, M., Słowikowska, A., et al. 2005a, ApJ, 633, 1101 (DFSRZ)

Dyks, J., Zhang, B., \& Gil, J. 2005b, ApJ, 626, L45

Edwards, R. T., Stappers, B. W., \& van Leeuwen, A. G. J. 2003, A\&A, 402, 321

Epstein, R. I. 1973, ApJ, 183, 593

Everett, J. E., \& Weisberg, J. M. 2001, ApJ, 553, 341

Fowler, L. A., \& Wright, G. A. E. 1982, A\&A, 109, 279

Gould, D. M., \& Lyne, A. G. 1998, MNRAS, 301, 235

Hankins, T. H., \& Cordes, J. M. 1981, ApJ, 249, 241

Hankins, T. H., \& Rankin, J. M. 2006, preprint

Harding, A. K., Usov V., \& Muslimov A. G. 2005, ApJ, 622, 531

Hirotani, K., Harding, A. K., \& Shibata, S. 2003, ApJ, 591, 334

Jenet, F. A., Anderson, S. B., Kaspi, V. M., et al. 1998, ApJ, 498, 365

Johnston, S., \& Romani, R. W. 2002, MNRAS, 332, 109

Komesaroff, M. M. 1970, Nature, 225, 612

Kramer, M., Lange, Ch., Lorimer, D. R., et al. 1999, ApJ, 526, 957

Kunzl, T., Lesch, H., Jessner, A., \& von Hoensbroech, A. 1998, ApJ, 505, L139

Kuzmin, A. D., Izvekova, V. A., Shitov, Yu. P., et al. 1998, A\&AS, 127, 355

Lange, Ch., Kramer, M., Wielebinski, R., \& Jessner, A. 1998, A\&A, 332, 111

Levinson, A., Melrose, D., Judge, A., \& Luo, Q. 2005, ApJ, 631, 456

Luo, Q., \& Melrose, D. B. 1992, MNRAS, 258, 616

Luo, Q., \& Melrose, D. B. 2006, MNRAS, 371, 1395

Lyubarski, Y. E., \& Petrova, S. A. 1998, A\&A, 337, 433

Malov, I. F., \& Machabeli, G. Z. 2001, ApJ, 554, 587

Manchester, R. N., \& Han, J. L. 2004, ApJ, 609, 354

Manchester, R. N., Hobbs, G. B., Teoh, A., \& Hobbs, M. 2005, AJ, 129, 1993

McConnell, D., Ables, J. G., Bailes, M., et al. 1996, MNRAS, 280, 331

McLaughlin, M. A., \& Rankin, J. M. 2004, MNRAS, 351, 808 (MR04)

Melrose, D. B. 1978, ApJ, 225, 557

Melrose, D. B. 2000, ASP Conf. Ser. 202, 721

Navarro, J., Manchester, R. N., Sandhu, J. S., et al. 1997, ApJ, 486, 1019 (NMSKB)

Nowakowski, L. 2003, Arecibo Newsletter, 36, 8

Perry, T. E., \& Lyne, A. G. 1985, MNRAS, 212, 489 (PL85)

Petrova, S. A. 2002, MNRAS, 336, 774

Phillips, J. A. 1990, ApJ, 361, L57

Radhakrishnan, V., \& Cooke, D. J. 1969, Astrophys. Lett., 3, 225

Rankin, J. M., \& Rathnasree, N. 1997, J. Astrophys. Astron., 18, 91

Romani, R. W., \& Yadigaroglu, I.-A. 1995, ApJ, 438, 314

Rowe, E. T. 1995, A\&A, 296, 275

Ruderman, M. A., \& Sutherland, P. G. 1975, ApJ, 196, 51

Rybicki, G. P., \& Lightman, A. P. 1979, Radiative processes in Astrophysics (New York: Wiley-Interscience)

Schopper, R., Ruhl, H., Kunzl, T. A., \& Lesch, H. 2002, MPE Report, 278, 193

Sturrock, P. A. 1971, ApJ, 164, 529

Takata, J., Shibata, S., Hirotani, K., \& Chang, H.-K. 2006, MNRAS, 366, 1310

Wang, F. Y.-H., Ruderman, M., Halpern, J. P., \& Zhu, T. 1998, ApJ, 438, 314

Weltevrede, P., Edwards, R. T., \& Stappers, B. W. 2006, A\&A, 445, 243

Wright, G. A. E. 2003, MNRAS, 344, 1041

Wright, G. A. E. 2004, MNRAS, 351, 813

Xilouris, K. M., Kramer, M., Jessner, A., et al. 1998, ApJ, 501, 286

Zheleznyakov, V. V., \& Shaposhnikov, V. E. 1979, Aust. J. Phys., 32, 49 


\section{Online Material}




\section{Appendix A: The pitch angle case}

The pitch angle case is discussed here for the sake of completeness, as well as in view of some positive features it has (see below). We begin with a short description of numerical results and then discuss how the model performs in confrontation with reality.

As the simplest choice of the electron's pitch-angle distribution $n_{\mathrm{e}}(\psi)$ we take the triangular shape:

$$
\begin{aligned}
& n_{\mathrm{e}}(\psi)=0 \text { for } \quad 0 \leq \psi \leq \psi_{\max }-\Delta \psi \\
& n_{\mathrm{e}}(\psi)=c_{\mathrm{in}}\left[\psi-\left(\psi_{\max }-\Delta \psi\right)\right] \Delta \psi^{-1} \\
& \text { for } \quad \psi_{\max }-\Delta \psi \leq \psi \leq \psi_{\max } \\
& n_{\mathrm{e}}(\psi)=c_{\text {out }}\left[\psi-\left(\psi_{\max }+\Delta \psi\right)\right]\left(-\Delta \psi^{-1}\right) \\
& \text { for } \quad \psi_{\max } \leq \psi \leq \psi_{\max }+\Delta \psi \\
& n_{\mathrm{e}}(\psi)=0 \quad \text { for } \quad \psi_{\max }+\Delta \psi \leq \psi \leq \pi
\end{aligned}
$$

where $c_{\text {in }}$ and $c_{\text {out }}$ can take the values of 0 or 1 and $\Delta \psi$ is the bottom width of the distribution. For $c_{\text {in }}=c_{\text {out }}=1$ the value of $n_{\mathrm{e}}(\psi)$ increases linearly from 0 at $\psi_{\max }-\Delta \psi$ up to 1 at $\psi_{\max }$, then drops linearly down to zero at $\psi_{\max }+\Delta \psi$. For $c_{\text {in }}=0$ (and $c_{\text {out }}=1$ ) only the outer part of the distribution is present with a sharp inner boundary at $\psi_{\max }$. For $c_{\text {out }}=0\left(\right.$ and $\left.c_{\text {in }}=1\right)$ the inner part of the distribution preserves.

Figure A.1 presents the look of notches for various types of the pitch-angle distribution $n_{\mathrm{e}}(\psi)$. In A.1a we have assumed a "two-sided" distribution $\left(c_{\text {in }}=c_{\text {out }}=1\right)$ with $\psi_{\max }=5^{\circ}$ and $\Delta \psi=0.2 \psi_{\max }$ which is an example of a general form of $n_{\mathrm{e}}(\psi)$ that is closely confined to $\psi_{\max }\left(\Delta \psi \ll \psi_{\max }\right)$. This case differs from the parallel acceleration case mainly in that $n_{\mathrm{e}}(\psi)$ covers much smaller solid angle (in comparison to $\theta_{\mathrm{R}}^{2}$ ) than the beam of non-negligible emission does in the $\boldsymbol{v} \| \boldsymbol{a}$ case. This has two consequences visible in Fig. A.1a: 1) the notches retain the high flux at the $\mathrm{W}$ center for the hole size larger than in the parallel acceleration case $\left(\rho_{\mathrm{h}} \lesssim 0.5 \theta_{\mathrm{R}}\right)$ because no radiation is emitted near the symmetry axis of the pitch-angle distribution $\left(\psi<4^{\circ}\right)$. 2) The W-shaped notches (with the unaffected central flux) are much deeper and reach the $\sim 20 \%$ depth observed for B1929+10. However, their shape becomes very distorted in comparison to the observed one. For $\rho_{\mathrm{h}}<0.4 \theta_{\mathrm{R}}$ the notches are narrow and separated by a flat plateau so that $\Delta \gg W$. For larger $\rho_{\mathrm{h}}$ the outer sides of the notches become rounded and much less steep than the inner ones that form the central maximum. All curves in panel a) correspond to the central viewing of the hole $(b=0)$ and $\gamma=10^{3}$. Figure A.1b presents result for the same pitch-angle distribution as in a) but $\rho_{\mathrm{h}}$ is fixed to $0.55 \theta_{\mathrm{R}}$ and $b$ is variable.

In Fig. A.1c $\psi_{\max }=\Delta \psi=5^{\circ}$, i.e. $n_{\mathrm{e}}(\psi)$ has a width comparable to $\psi_{\text {max }}$. As before, $c_{\text {in }}=c_{\text {out }}=1$, i.e. the distribution is symmetrical with respect to $\psi_{\max }$. This shape is qualitatively similar to the shape of the radiation beam for the parallel acceleration case. Therefore the resulting notches look alike. The result is for $b=0$.

Figure A.1d presents results for the inner pitch-angle distribution ( $c_{\text {out }}=0$ in Eq. (A.1)) in the case of $b=0$ and variable $\rho_{\mathrm{h}}$. Here the separation between the notches is in general different from their width: $\Delta>W$ for $\rho_{\mathrm{h}} \lesssim 0.2 \theta_{\mathrm{R}}$ whereas $\Delta<W$ for $\rho_{\mathrm{h}} \gtrsim 0.3 \theta_{\mathrm{R}}$. The notches have peculiar shape.

Figure A.1e is for the outer $n_{\mathrm{e}}(\psi)$ distribution (Eq. (A.1) with $\left.c_{\text {in }}=0\right)$. The shape of notches is again unlike the observed one. The W center is flat for $\rho_{\mathrm{h}} \lesssim 0.5 \theta_{\mathrm{R}}$ or has a dip for $\rho_{\mathrm{h}} \simeq 0.75 \theta_{\mathrm{R}}$ and the notches look strange.

Figure A.1f is for the pitch-angle distribution considered by Erber (1973): $n_{\mathrm{e}} \propto \psi \exp \left[-\psi^{2} / \psi_{\max }^{2}\right]$. Its general properties are similar to the case shown in Fig. A.1c $\left(\Delta \psi \sim \psi_{\max }\right)$ and therefore the resulting notches have similar shape, close to the observed one.

The general property that is common for all the cases is that for very small $\rho_{\mathrm{h}}$ the hole acts as a delta function and the resulting notches have the same shape as $n_{\mathrm{e}}(\psi)$. Therefore, the notches have sharp minima (and are very shallow) whenever $\rho_{\mathrm{h}} \ll \psi_{\max }$ (topmost curve(s) in panels a, c, d, and e). For larger $\rho_{\mathrm{h}}$ the minima become oblate in all cases with the exception of the inner $n_{\mathrm{e}}(\psi)$ case (panel d). It is worth emphasizing that the observed notches appear much sharper than they really are on most plots that show full rotation period (cf. top line in Fig. 1a with exactly the same profile shown in Fig. 2a).

A few points on confrontation of the model with the data:

The conclusions 1 to 3 in Sect. 5 are valid for both the parallel acceleration case as well as for a (quite numerous) class of the pitch-angle distributions that fulfill $\psi_{\max } \sim \Delta \psi$.

The initial pitch angle of electron-positron pairs created through one-photon absorption is also inversely proportional to $\gamma$. This is because photons propagating at small angles $\psi$ relative to $\boldsymbol{B}$ need to have larger energy to produce pairs $\left[\left(\epsilon / m c^{2}\right)\left(B / B_{\mathrm{Q}}\right) \sin \psi \approx 2 / 15\right.$, Ruderman \& Sutherland 1975] and the pair components tend to share the energy $\epsilon$ of a parent photon equally ( $\gamma m c^{2} \approx \epsilon / 2$, Daugherty \& Harding 1983), which leads to

$\gamma \approx \frac{1}{15}\left(\frac{B}{B_{\mathrm{Q}}}\right)^{-1} \frac{1}{\sin \psi}$

Afterwards $\psi$ undergoes strong evolution but its value remains anticorrelated with $\gamma$ (see Fig. 2 in Harding et al. 2005).

The distribution of pitch angles may be considered fragile and susceptible to disturbances, which is consistent with the probable variability of the notches and pedestal emission on a timescale of days.

The low $|\boldsymbol{B}|$ required for the large $\psi_{\max }$ is consistent with the observed location of the pedestal emission (far from MP in B1929+10 and B0950+08) as well as with the presence of notches in J0437-4715. The magnetar does not seem to fit into the picture, although we do not know how large the radio emission altitude in XTE J1810-197 might be.

Since we do not see how the relation $\Delta \propto v_{\mathrm{obs}}^{-1 / 2}$ could arise in the scenario of the favored pitch angle, we consider it less natural than the $\boldsymbol{a} \| \boldsymbol{v}$ case.

\section{Appendix B: Single pulse visibility of double features}

\section{B.1. The quasi-steady but nonuniform source at fixed altitude} (drifting spots)

As can be seen in Fig. 3b the notches can be observed in full form in single pulse data only when the absorber is nearlysimultaneously (or: for a sufficiently long period of time $>\Delta t_{\text {rot }}$ ) illuminated from various directions by a sufficiently large part of the emission region (extending at least between the two points marked with the small letters "d" in Fig. 3b). In the hole case a nearly-simultaneous emission around the hole is required (or at least on opposite sides of the hole.) For a random distribution of sparks (emission spots) of angular radius $\rho_{\text {sprk }} \lesssim \theta_{\mathrm{R}}$ one can observe only a part of the notch feature (a shoulder or dip) or no absorption feature at all. The latter should frequently occur because there are a great deal of points on the emission region (e.g. marked with "b" in Fig. 3) that contribute unobscured radiation at the pulse phase of the notches. The phase at which 

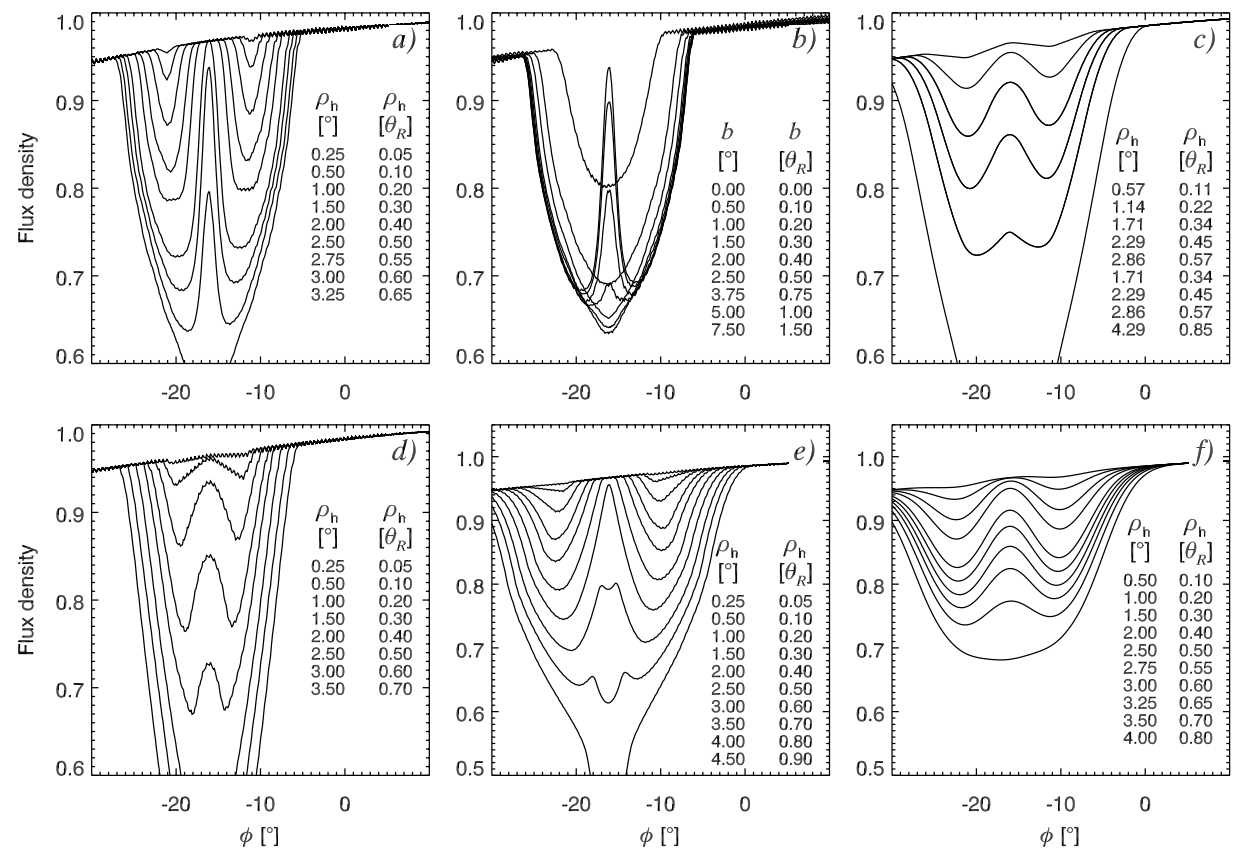

Fig. A.1. Modelled double notches calculated for various pitch-angle distributions with a fixed $\gamma=10^{3}$ and $\psi_{\max }=$ $5^{\circ}$. A hole in the emission region is assumed. For details see text.

the radiation is detected is marked also with " $b$ " just above the schematic pulse profiles at the top of Fig. 3. In this scenario it would be natural to see no single pulse with the double notches. There would also be lots of single emission features because of contribution of points located outside of the plane of Fig. 3 at angular distance $\sim \theta_{\mathrm{R}}$ from the line of sight traverse. One would have also seen, however, a great number of double subpulses corresponding to the sightline cut through the elementary cones, which is not observed. The model just discussed is thus excluded by the observed single pulse properties of pulsar radio emission. In principle, however, such kind of emitter could be a part of magnetosphere that stays at a fixed altitude and is steadily refreshed by electrons that keep flowing through it.

\section{B.2. Bunches of electrons outflowing at relativistic speeds}

A much more realistic model for the basic emission units in pulsar magnetosphere is based on short living bunches of electrons that outflow at relativistic speeds $(\gamma \sim 10)$. Such sources are localized both in the radial and horizontal direction, but their radial coordinate increases at nearly the speed of light $\mathrm{d} r / \mathrm{d} t=0.995 c$ for $\gamma=10$. The chance to observe both sides of the elementary emission cone from a single bunch fully depends on whether the bunch can survive for sufficiently long period of time, namely the time interval $t_{\mathrm{t}}-t_{\mathrm{l}}$ needed for the cone to expose its other side to the observer.

The source (bunch) is now approaching the observer (and trying to catch up with the photons) with $\gamma \sim 10$ so that its radial distance changes according to $r \simeq c t+r_{0}$. For definiteness, hereafter we assume that the first side of the cone is spotted by the observer at time $t_{0}=t_{1}=0$ and at the radial distance $r_{0}=r\left(t_{0}\right) \sim R_{\mathrm{NS}}$ that will be neglected because $r_{0} \ll \Delta r_{\text {rot }}$ for most periods we are interested in. Let the azimuth of the emission direction on the observed side of the cone is $\phi_{1}=0$ (in the observer's frame OF), which is also the fixed azimuth of the line of sight. For a bunch that flows along the dipole axis, the azimuth of the other side of the cone will change according to

$\phi_{\mathrm{t}} \simeq 2 \theta_{\mathrm{R}}-\Omega t-\frac{r}{R_{\mathrm{lc}}} \simeq \Delta-2 \frac{c t}{R_{\mathrm{lc}}}$, where $2 \theta_{\mathrm{R}}$ is the initial value, $\Omega t$ takes into account the rotation of the dipole and $r / R_{\mathrm{lc}}$ takes into account the forward projection of radiation caused by the aberration effect in the limit $r_{0} \ll r$ (e.g. Dyks et al. 2004b). The observer can see the trailing side of the cone $\left(\phi_{\mathrm{t}}=0\right)$ after time $t=P \Delta /(4 \pi)$ which is two times smaller $\left(3 \times 10^{-5}-10^{-3} \mathrm{~s}\right)$ than $\Delta t_{\text {rot }}$ given by Eq. (6) and corresponds to light travel lengthscales of $(1-50) \times 10^{6} \mathrm{~cm}$. The scales are much longer than predicted/considered in some models of radio coherency, e.g. $\Delta r \sim$ a few $\times 10^{5} \mathrm{~cm}$ in Schopper et al. (2002) or in the perpendicular acceleration model of Fung \& Kuijpers (2004).

Had, however, the bunch survived for the time $t_{\mathrm{t}}-t_{1}$ without any significant evolution (to maintain its original hollow cone emission beam) then the trailing side of the cone would not be observed $\sim 5^{\circ}$ after the leading side, but much sooner. Because the bunch propagates towards the observer with the speed $v$ close to $c$, the radiation in the trailing side lags the radio waves from the leading side by $\Delta r \simeq c \Delta t-v \Delta t \simeq c \Delta t /\left(2 \gamma^{2}\right)$. The trailing radio waves are then detected just after the leading side radio waves:

$\Delta t_{\mathrm{obs}}=\frac{t_{\mathrm{t}}-t_{1}}{2 \gamma^{2}}=5 \times 10^{-8} \mathrm{~s} \frac{t_{\mathrm{t}}-t_{1}}{10^{-5} \mathrm{~s}}\left(\frac{\gamma}{10}\right)^{-2}$

so that $\Omega \Delta t_{\mathrm{obs}} \ll \Delta$. To detect both sides of the hollow cone from a single bunch or particle, one would therefore need to look for double emission features on the timescales of nanoseconds to milliseconds. The bunch, however, and the physical conditions around it could not evolve significantly during the time $t_{\mathrm{t}}-t_{1} \sim$ $10^{-5}-10^{-3} \mathrm{~s}$ which may be impossible to satisfy.

We therefore conclude that the direct observation of the elementary hollow cone in the single pulse data is extremely improbable, if not impossible. In any case, in the outflowing bunch scenario the $\sim 5^{\circ}$ opening angle of the cone would correspond (in single pulse data) to double emission spikes separated by $\sim 0.01^{\circ}$. The $5^{\circ}$ separation is visible in the averaged pulse profiles because the trailing notch (or the trailing maximum in Fig. 7) is created by radio waves emitted from roughly the same altitude as those associated with the leading notch/maximum, but emitted later by a different bunch of electrons. Therefore, the only sign 
of the double notches in the single pulse data will be the less frequent appearance of single-looking, narrow emission spikes that are normally observed in the high time resolution data.

\section{B.3. The role of B-field line curvature}

The above estimates have been done for the special case of the dipole axis, but the curvature of magnetic field lines can drag the other side of the hollow cone into the observer's view. On the leading side of the dipole axis, the Eq. (B.1) becomes:

$\phi_{\mathrm{t}} \simeq 2 \theta_{\mathrm{R}}-\Omega t-\frac{r}{R_{\mathrm{lc}}}-\frac{r}{\rho_{\mathrm{crv}}}$

where $\rho_{\text {crv }}$ in the last term is the radius of curvature of magnetic field lines. The second side of the hollow cone is directed towards the observer after time

$c t \simeq \frac{\Delta}{\rho_{\mathrm{crv}}^{-1}+2 R_{\mathrm{lc}}^{-1}}$.

At the last open field lines $\rho_{\text {crv }} \simeq(4 / 3)\left(r R_{\mathrm{lc}}\right)^{1 / 2}=9.2 \times$ $10^{7}\left(P r_{6}\right)^{1 / 2}$ where $r_{6}=r /\left(10^{6} \mathrm{~cm}\right)$. For the considered range of periods (5-250 ms) and $r \sim 10^{6} \mathrm{~cm}$ the resulting lengthscales are $(0.5-5) \times 10^{6} \mathrm{~cm}$ and become larger for increasing $r$, e.g. $(0.7-12) \times 10^{6} \mathrm{~cm}$ for $r_{6}=10$. The lower limit of the scales (that refers to the $5 \mathrm{~ms}$ period) is still of the order of $R_{\mathrm{NS}}$. The scale has decreased considerably only for the normal, long period pulsars.

On the trailing side of the dipole axis the sign of the last term in Eq. (B.3) is positive and the equation has no positive solutions for $t$ if $\rho_{\mathrm{crv}}^{-1}>2 R_{\mathrm{lc}}^{-1}$, e.g. near the edge of the polar cap. This simply means that the combined effects of rotation and aberration (2nd and 3rd terms in Eq. (B.3)) are too weak to compensate for the backward bending of the $\boldsymbol{B}$-field lines, i.e. the field line's curvature drags the beam away from the observer's line of sight. A full cut through the beam is possible only when the observer sees the trailing side of the cone first. Appropriate change of signs in (B.3) gives then

$c t \simeq \frac{\Delta}{\rho_{\mathrm{crv}}^{-1}-2 R_{\mathrm{lc}}^{-1}}$

which gives timescales only slightly larger than on the leading side because $\rho_{\text {crv }} \ll R_{\text {lc }} / 2$ in the region where we make estimates (low altitudes, close to the last open field lines). An interesting solution of (B.5) corresponds to $\rho_{\text {crv }}=R_{\mathrm{lc}} / 2$ (field lines slightly on the trailing side of the dipole axis) for which $t=\infty$. This is the case in which the beam does not rotates in the obsever's frame, i.e. the field line curvature fully compensates aberration and rotation. The observer can see the leading side of the beam all the time as the bunch propagates upwards (caustic pile up of radiation near a fixed phase in pulse profile), actually up to the altitude above which our simple approximations (B.1)-(B.5) break down.

In the curved magnetic field lines the hollow cone emission caused by the parallel acceleration becomes dominated by the curvature emission at frequencies $v \lesssim c \gamma^{3} /\left(2 \pi \rho_{\text {crv }}\right)$ (Melrose 1978). For the range of $P=5-250 \mathrm{~ms}$ and $\rho_{\text {crv }}$ at the polar cap rim the curvature radiation declines above $0.1-0.7 \mathrm{MHz}$ which is well below the frequencies at which the notches are observed. 